\title{
Angular Momentum Mixing in Single Flavor Color Superconductivity with Transverse Pairing
}

\author{
Bo Feng, ${ }^{1, *}$ De-fu Hou, ${ }^{1, \oplus}$ and Hai-cang Ren ${ }^{2,1,}$, \\ ${ }^{1}$ Institute of Particle Physics, Huazhong Normal University, Wuhan, 430079, China \\ ${ }^{2}$ Physics Department, The Rockefeller University, 1230 York Avenue, New York, NY 10021-6399
}

(Dated: November 2, 2018)

\begin{abstract}
Because of the equal strength of the pairing potential mediated by one-gluon exchange for all partial waves to the leading order QCD running coupling constant and the nonlinearity of the gap equation, the non-spherical pairing in single flavor color superconductivity(CSC) can not be restricted in a single non-s-wave channel and the mixing among different angular momenta will occur. In this paper, we examine the angular momentum mixing in single flavor CSC with transverse pairing, in which the pairing quarks have opposite helicity. We find that the free energy of all nonspherical pairing states are lowered by angular momentum mixing compared with that contain p-wave only. But the amount of the free energy drop is numerically small. Consequently the most stable pairing state that respect the time reversal invariance remains the spherical CSL.
\end{abstract}

PACS numbers: 12.38.Aw, 24.85.+p, 11.15.Ex

\section{INTRODUCTION}

Color superconductivity(CSC) is expected to be the ground state in a quark matter of sufficiently high baryon density but low temperature [1, 2, 3, 4, 5, 6, 7]. The most favorable pairing channel in the chiral limit is between quarks of different flavors. At the baryon density accessible in a compact star, however, the nonzero strange quark mass induces a significant mismatch between Fermi momenta of different flavors that suppresses the phase space available for pairing. A lot of exotic CSC states or mixed states have been proposed in the literature [8, 9, 10, 11, 12]. But the result of their competition remains under debate.

Since the single flavor pairing is free from the Fermi momentum mismatch, it has been considered as an interesting alternative in this circumstance. The corresponding gap function in this case should contain higher total angular momentum as required by Pauli principle, the obvious alternative is to consider the p-wave pairing $(J=1)[13$, 14, 15, [16, 17, 18]. A large variety of pairing structures of the single flavor CSC has been discussed in the literature 13, 14]. Except for the color-spin-locked(CSL) state, none of the energy gaps is spherical. All these works restrict the angular dependence of a non-spherical gap within a single partial wave, $p$-wave, which is valid if the pairing potential consists of the $p$-channel only, such as that in the superfluid ${ }^{3} \mathrm{He}$. The forward singularity of diquark scattering in QCD, however, brings in all angular momentum channels to the pairing potential. Because of the nonlinearity of the gap equation, all partial waves contribute to a non-spherical energy gap at the same order of the QCD running coupling. In a previous paper [19], we examined this angular momentum mixing effect in the non-spherical CSC with longitudinal pairing, in which the two pairing quarks carry the same helicity. We found that the energy gap ( gap function evaluated at zero Matsubara energy ) takes the form

$$
\phi(0, \hat{\mathbf{p}})=\frac{2048 \sqrt{2} \pi^{4} \mu}{N_{f}^{5 / 2} g^{5}} e^{-\frac{3 \pi^{2}}{\sqrt{2} g}-\frac{\pi^{2}+4}{8}} f(\hat{\mathbf{p}})
$$

where $\mu$ is the chemical potential, $\hat{\mathbf{p}}$ is the direction of the relative momentum in a Cooper pair and the function $f(\hat{\mathbf{p}})$ is an infinite series in spherical harmonics of all $J$ 's. In particular, we find

$$
f(\hat{\mathbf{p}})=e^{1 / 3} e^{-6}\left[0.9866 P_{1}(\cos \theta)-0.0673 P_{3}(\cos \theta)+0.0214 P_{5}(\cos \theta)+\ldots\right]
$$

in contrast with $f(\hat{\mathbf{p}})=e^{1 / 3} e^{-6} P_{1}(\cos \theta)$ reported in the literature for the polar phase. The contributions from higher partial waves, however, are numerically small. The same effect is expected for the transverse pairing and the parallel analysis will be carried out in this paper.

\footnotetext{
*Electronic address: fengbo@iopp. ccnu. edu.cn

${ }^{\dagger}$ Electronic address: hdf@iopp. ccnu. edu.cn

‡Electronic address: ren@mail.rockefeller.edu
} 
The free energy density of the superconductivity with angular momentum mixing will be lowered relative to that containing p-wave pairing only. In the toy model considered in [19], which maximizes the forward singularity, the magnitude of the condensation energy is increased by a factor of 1.54. Without including the angular momentum mixing, the gaining factor of the magnitude of the condensation energy in CSC by forming the spherical CSL state compared with the non-spherical polar state is 1.54 for the longitudinal pairing and 1.14 for the transverse pairing [14]. This raises the issue to recalculate the condensation energy of various non-spherical pairing states of the single-flavor CSC in the light of the angular momentum mixing and to compare the result with that of the spherical CSL. So we did in previous works [19] [20] for the polar and A phases of the longitudinal pairing. The formulation we developed can be carried over readily to the polar and A phases of the transverse pairing and the parallel analysis is made in this work. We found that, like the longitudinal pairing case, the amount of mixing is numerically small so the slightly reduced free energy of the polar and A phases is not low enough to compete with the spherical CSL phase.

This paper is organized as follows. In Sec. II, we examine the quark propagator with transverse pairing. The condensation energy density and the gap equation will be obtained from the CJT effective action in Section III. The gap equation will be reduced to an nonlinear integral equation for the angular dependence with zero and nonzero azimuthal quantum number in Section IV and their numerical solutions will be given there as well. We conclude in Sec. V. Some technical details about the full quark propagators and the relevant spherical harmonics are deferred to Appendices A and B. Our convention for the metric tensor is $g_{\mu \nu}=\operatorname{diag}\{1,-1,-1,-1\}$. Our units are $\hbar=c=k_{B}=1$ and 4 -vectors are denoted by capital letters, $K \equiv K^{\mu}=(i \nu, \mathbf{k})$ with $\nu$ the Matsubara energy, which becomes continuous at zero temperature, $T=0$.

\section{THE QUARK PROPAGATOR FOR TRANSVERSE PAIRING}

Though the general structure of the quark propagator in CSC state has been discussed extensively in the literature [21], the formulation we developed below is particularly suitable to explore the angular momentum mixing in a transverse pairing state.

We start with the thermal Nambu-Gorkov quark propagator $\mathcal{S}(X)$, defined as

$$
S_{\alpha \beta}(X)=<\mathcal{T}\left[\Psi_{\alpha}(X) \bar{\Psi}_{\beta}(0)\right]>=T \sum_{\nu} \int \frac{d^{3} \mathbf{p}}{(2 \pi)^{3}} e^{i P \cdot X} \mathcal{S}(P)
$$

where $\Psi$ and $\bar{\Psi}$ are the Nambu-Gorkov(NG) spinors, $P=(i \nu, \mathbf{p}), P \cdot X=-\nu \tau-\mathbf{p} \cdot \mathbf{x}$ with $\nu$ the Matsubara energy and $\tau$ the Euclidean time, $\mathcal{T}$ imposes the Euclidean time ordering and $(\alpha, \beta)$ run over the NG, Dirac and color indexes. At zero temperature, $T \sum_{\nu}=\int_{-\infty}^{\infty} \frac{d \nu}{2 \pi}$. The NG spinors are defined by

$$
\Psi=\left(\begin{array}{c}
\psi \\
\psi_{C}
\end{array}\right), \quad \bar{\Psi}=\left(\bar{\psi}, \bar{\psi}_{C}\right)
$$

where, $\psi$ and $\bar{\psi}$ are written in terms of the ordinary quark spinors, $\psi_{C}=C \tilde{\bar{\psi}}$ and $\bar{\psi}_{C}=\tilde{\psi} C$ are the charge-conjugate spinors with the tilde standing for the transpose, and $C \equiv i \gamma^{2} \gamma^{0}$. It follows from the definition (3) and the selfconjugation relation $\Psi=\mathcal{C} \Psi^{*}$ with

$$
\mathcal{C}=\left(\begin{array}{cc}
0 & i \gamma_{2} \\
i \gamma_{2} & 0
\end{array}\right)
$$

that

$$
S^{*}(P)=\mathcal{C}^{\dagger} S(\bar{P}) \mathcal{C}
$$

with $\bar{P}=(i \nu,-\mathbf{p})$ and

$$
\tilde{\mathcal{S}}(P)=\Gamma_{0} \mathcal{C S}(-P) \Gamma_{0} \mathcal{C}
$$

with

$$
\Gamma_{0}=\left(\begin{array}{cc}
\gamma_{0} & 0 \\
0 & \gamma_{0}
\end{array}\right)
$$

The relation (6) was proved in [22] and the proof of (77) proceeds similarly. The NG structure of the inverse propagator is

$$
S^{-1}(P) \equiv\left(\begin{array}{cc}
K^{+}(P) & \Phi^{-}(P) \\
\Phi^{+}(P) & K^{-}(P)
\end{array}\right)
$$


where each block is a $4 \times 4$ matrix with respect to Dirac indexes and a $3 \times 3$ matrix in color indexes. We shall focus on the off-diagonal blocks $\Phi^{ \pm}(P)$ below.

It is convenient to work in the chiral representation where

$$
\psi=\left(\begin{array}{c}
\chi_{R} \\
\chi_{L}
\end{array}\right), \quad \bar{\psi}=\left(\chi_{L}^{+}, \chi_{R}^{+}\right)
$$

and their charge-conjugate counterparts

$$
\psi_{C}=\left(\begin{array}{c}
i \sigma^{2} \chi_{L}^{*} \\
-i \sigma^{2} \chi_{R}^{*}
\end{array}\right), \quad \bar{\psi}_{C}=\left(i \tilde{\chi}_{R} \sigma^{2},-i \tilde{\chi}_{L} \sigma^{2}\right),
$$

with $\chi_{L}\left(\chi_{R}\right)$ the two-component left-handed(right-handed spinors) and $\sigma^{2}$ the second Pauli matrix. The matrix $\Phi^{ \pm}(P)$ are decomposed accordingly. For example,

$$
\Phi^{-}(P) \equiv\left(\begin{array}{cc}
\Phi_{++}^{-}(P) & \Phi_{+-}^{-}(P) \\
\Phi_{-+}^{-}(P) & \Phi_{--}^{-}(P)
\end{array}\right) \text {. }
$$

with each block a $2 \times 2$ matrix with respect to spinor indexes. For transverse pairing between different chiralities, $\Phi_{++}^{ \pm}(P)=\Phi_{--}^{ \pm}(P)=0$. Next, we consider the off-diagonal block $\Phi_{+-}^{-}(P)$ and write

$$
\Phi_{+-}^{-}(P)=\varphi(P)+\varphi_{0}(P) \boldsymbol{\sigma} \cdot \hat{\mathbf{p}}+\varphi_{+}(P) \boldsymbol{\sigma} \cdot \hat{\mathbf{e}}_{+}(\hat{\mathbf{p}})+\varphi_{-}(P) \boldsymbol{\sigma} \cdot \hat{\mathbf{e}}_{-}(\hat{\mathbf{p}})
$$

where the circular basis $\hat{\mathbf{e}}_{ \pm}(\hat{\mathbf{p}})=\frac{1}{\sqrt{2}}(\hat{\boldsymbol{\theta}} \pm i \hat{\boldsymbol{\varphi}})$ with $\hat{\boldsymbol{\theta}}$ and $\hat{\boldsymbol{\varphi}}$ the unit vectors of the spherical coordinates of $\mathbf{p}$. We have

$$
\begin{aligned}
& \hat{\mathbf{p}}=(\sin \theta \cos \varphi, \sin \theta \sin \varphi, \cos \theta) \\
& \hat{\boldsymbol{\theta}}=(\cos \theta \cos \varphi, \cos \theta \sin \varphi,-\sin \theta) \\
& \hat{\boldsymbol{\varphi}}=(-\sin \varphi, \cos \varphi, 0), \\
& \hat{\mathbf{e}}_{+}(-\hat{\mathbf{p}})=\hat{\mathbf{e}}_{-}(\hat{\mathbf{p}}) \quad \hat{\mathbf{e}}_{+}(\hat{\mathbf{p}})^{*}=\hat{\mathbf{e}}_{-}(\hat{\mathbf{p}}),
\end{aligned}
$$

and

$$
\hat{\mathbf{p}} \times \hat{\mathbf{e}}_{ \pm}(\hat{\mathbf{p}})=\mp i \hat{\mathbf{e}}_{ \pm}(\hat{\mathbf{p}}) .
$$

Performing a rotation about $\mathbf{p}$ by an angle $\gamma$, we find

$$
\Phi_{+-}^{-}(P) \rightarrow e^{-\frac{i}{2} \gamma \boldsymbol{\sigma} \cdot \hat{\mathbf{p}}} \Phi_{+-}^{-}(P) e^{\frac{i}{2} \gamma \boldsymbol{\sigma} \cdot \hat{\mathbf{p}}}=\varphi(P)+\varphi_{0}(P) \boldsymbol{\sigma} \cdot \hat{\mathbf{p}}+e^{-i \gamma} \varphi_{+}(P) \boldsymbol{\sigma} \cdot \hat{\mathbf{e}}_{+}(\hat{\mathbf{p}})+e^{i \gamma} \varphi_{-}(P) \boldsymbol{\sigma} \cdot \hat{\mathbf{e}}_{-}(\hat{\mathbf{p}})
$$

For a Copper pair of two quarks or two antiquarks of opposite helicity, the projection of its angular momentum in the direction $\mathbf{p}$ has to be \pm 1 , which implies that $\varphi(P)=\varphi_{0}(P)=0$. Up to now, the coefficients $\varphi$ 's remain $3 \times 3$ antisymmetric matrices in color space. For the polar phase and A phase considered in this work, we set

$$
\Phi_{+-}^{-}(P)=\boldsymbol{\sigma} \cdot \boldsymbol{\Psi}(P) \lambda_{2}
$$

where

$$
\boldsymbol{\Psi}(P)=\phi_{+}(P) \hat{\mathbf{e}}_{+}(\hat{\mathbf{p}})+\phi_{-}(P) \hat{\mathbf{e}}_{-}(\hat{\mathbf{p}}) .
$$

and $\lambda_{2}$ is the second Gell-Mann matrix.

The Dirac structure of other off-diagonal elements in Eq.(9) can be deduced from the relations (6) and (7). We now could write down the inverse full quark propagator in chiral representation using the Dyson-Schwinger equation and ignoring the contribution from the wave-function renormalization, and reads

$$
S^{-1}(P)=\left(\begin{array}{cccc}
0 & i \nu+\mu-\boldsymbol{\sigma} \cdot \mathbf{p} & 0 & \lambda_{2} \boldsymbol{\sigma} \cdot \boldsymbol{\Psi}(P) \\
i \nu+\mu+\boldsymbol{\sigma} \cdot \mathbf{p} & 0 & -\lambda_{2} \boldsymbol{\sigma} \cdot \boldsymbol{\Psi}(-P) & 0 \\
0 & \lambda_{2} \boldsymbol{\sigma} \cdot \boldsymbol{\Psi}^{*}(\bar{P}) & 0 & i \nu-\mu-\boldsymbol{\sigma} \cdot \mathbf{p} \\
-\lambda_{2} \boldsymbol{\sigma} \cdot \boldsymbol{\Psi}^{*}(-\bar{P}) & 0 & i \nu-\mu+\boldsymbol{\sigma} \cdot \mathbf{p} & 0
\end{array}\right)
$$


Inverting the matrix (20) (see the appendix A for details), we find that

$$
S(P)=\left(\begin{array}{cccc}
S_{11} & S_{12} & S_{13} & S_{14} \\
S_{21} & S_{22} & S_{23} & S_{24} \\
S_{31} & S_{32} & S_{33} & S_{34} \\
S_{41} & S_{42} & S_{43} & S_{44}
\end{array}\right)
$$

with

$$
\begin{aligned}
& S_{11}=S_{13}=S_{22}=S_{24}=S_{31}=S_{33}=S_{42}=S_{44}=0 \\
& S_{12}=-\frac{i \nu-(p+\mu)}{\nu^{2}+(p+\mu)^{2}+2 \lambda_{2}^{2} \phi_{-}(-P) \phi_{-}^{*}(-\bar{P})} \frac{1+\boldsymbol{\sigma} \cdot \hat{\mathbf{p}}}{2}-\frac{i \nu+(p-\mu)}{\nu^{2}+(p-\mu)^{2}+2 \lambda_{2}^{2} \phi_{+}(-P) \phi_{+}^{*}(-\bar{P})} \frac{1-\boldsymbol{\sigma} \cdot \hat{\mathbf{p}}}{2} \\
& S_{14}=-\frac{\lambda_{2} \boldsymbol{\sigma} \cdot \hat{\mathbf{e}}_{+}(\hat{\mathbf{p}}) \phi_{-}(-P)}{\nu^{2}+(p+\mu)^{2}+2 \lambda_{2}^{2} \phi_{-}(-P) \phi_{-}^{*}(-\bar{P})} \frac{1-\boldsymbol{\sigma} \cdot \hat{\mathbf{p}}}{2}-\frac{\lambda_{2} \boldsymbol{\sigma} \cdot \hat{\mathbf{e}}_{-}(\hat{\mathbf{p}}) \phi_{+}(-P)}{\nu^{2}+(p-\mu)^{2}+2 \lambda_{2}^{2} \phi_{+}(-P) \phi_{+}^{*}(-\bar{P})} \frac{1+\boldsymbol{\sigma} \cdot \hat{\mathbf{p}}}{2} \\
& S_{21}=-\frac{i \nu-(p+\mu)}{\nu^{2}+(p+\mu)^{2}+2 \lambda_{2}^{2} \phi_{-}(P) \phi_{-}^{*}(\bar{P})} \frac{1-\boldsymbol{\sigma} \cdot \hat{\mathbf{p}}}{2}-\frac{i \nu+(p-\mu)}{\nu^{2}+(p-\mu)^{2}+2 \lambda_{2}^{2} \phi_{+}(P) \phi_{+}^{*}(\bar{P})} \frac{1+\boldsymbol{\sigma} \cdot \hat{\mathbf{p}}}{2} \\
& S_{23}=\frac{\lambda_{2} \boldsymbol{\sigma} \cdot \hat{\mathbf{e}}_{-}(\hat{\mathbf{p}}) \phi_{-}(P)}{\nu^{2}+(p+\mu)^{2}+2 \lambda_{2}^{2} \phi_{-}(P) \phi_{-}^{*}(\bar{P})} \frac{1+\boldsymbol{\sigma} \cdot \hat{\mathbf{p}}}{2}+\frac{\lambda_{2} \boldsymbol{\sigma} \cdot \hat{\mathbf{e}}_{+}(\hat{\mathbf{p}}) \phi_{+}(P)}{\nu^{2}+(p-\mu)^{2}+2 \lambda_{2}^{2} \phi_{+}(P) \phi_{+}^{*}(\bar{P})} \frac{1-\boldsymbol{\sigma} \cdot \hat{\mathbf{p}}}{2} \\
& S_{32}=-\frac{\lambda_{2} \boldsymbol{\sigma} \cdot \hat{\mathbf{e}}_{-}(\hat{\mathbf{p}}) \phi_{-}^{*}(-\bar{P})}{\nu^{2}+(p+\mu)^{2}+2 \lambda_{2}^{2} \phi_{-}(-P) \phi_{-}^{*}(-\bar{P})} \frac{1+\boldsymbol{\sigma} \cdot \hat{\mathbf{p}}}{2}-\frac{\lambda_{2} \boldsymbol{\sigma} \cdot \hat{\mathbf{e}}_{+}(\hat{\mathbf{p}}) \phi_{+}^{*}(-\bar{P})}{\nu^{2}+(p-\mu)^{2}+2 \lambda_{2}^{2} \phi_{+}(-P) \phi_{+}^{*}(-\bar{P})} \frac{1-\boldsymbol{\sigma} \cdot \hat{\mathbf{p}}}{2} \\
& S_{34}=-\frac{i \nu+(p+\mu)}{\nu^{2}+(p+\mu)^{2}+2 \lambda_{2}^{2} \phi_{-}(-P) \phi_{-}^{*}(-\bar{P})} \frac{1-\boldsymbol{\sigma} \cdot \hat{\mathbf{p}}}{2}-\frac{i \nu-(p-\mu)}{\nu^{2}+(p-\mu)^{2}+2 \lambda_{2}^{2} \phi_{+}(-P) \phi_{+}^{*}(-\bar{P})} \frac{1+\boldsymbol{\sigma} \cdot \hat{\mathbf{p}}}{2} \\
& S_{41}=\frac{\lambda_{2} \boldsymbol{\sigma} \cdot \hat{\mathbf{e}}_{+}(\hat{\mathbf{p}}) \phi_{-}^{*}(\bar{P})}{\nu^{2}+(p+\mu)^{2}+2 \lambda_{2}^{2} \phi_{-}(P) \phi_{-}^{*}(\bar{P})} \frac{1-\boldsymbol{\sigma} \cdot \hat{\mathbf{p}}}{2}+\frac{\lambda_{2} \boldsymbol{\sigma} \cdot \hat{\mathbf{e}}_{-}(\hat{\mathbf{p}}) \phi_{+}^{*}(\bar{P})}{\nu^{2}+(p-\mu)^{2}+2 \lambda_{2}^{2} \phi_{+}(P) \phi_{+}^{*}(\bar{P})} \frac{1+\boldsymbol{\sigma} \cdot \hat{\mathbf{p}}}{2} \\
& S_{43}=-\frac{i \nu+(p+\mu)}{\nu^{2}+(p+\mu)^{2}+2 \lambda_{2}^{2} \phi_{-}(P) \phi_{-}^{*}(\bar{P})} \frac{1+\boldsymbol{\sigma} \cdot \hat{\mathbf{p}}}{2}-\frac{i \nu-(p-\mu)}{\nu^{2}+(p-\mu)^{2}+2 \lambda_{2}^{2} \phi_{+}(P) \phi_{+}^{*}(\bar{P})} \frac{1-\boldsymbol{\sigma} \cdot \hat{\mathbf{p}}}{2} \text {. }
\end{aligned}
$$

We notice that $\phi_{+}$associates with particles and $\phi_{-}$with antiparticles. The is because we designate implicitly the momentum $\mathbf{p}$ to the quark of positive helicity in a Cooper pair. Since the contribution from the Cooper pairs of antiquarks goes beyond the subleading order, we shall approximate $\phi_{-}(P) \simeq 0$ and introduce the gap function $\phi(\nu, \mathbf{p})$ via

$$
\phi_{+}(P) \equiv \frac{1}{\sqrt{2}} \phi(\nu, \mathbf{p})
$$

As is the same with 2SC, the solution to the gap equation gives rise to an even function in the Matsubara energy, we have $\phi_{+}(-P)=\frac{1}{\sqrt{2}} \phi(\nu,-\mathbf{p}), \phi_{+}(\bar{P})=\frac{1}{\sqrt{2}} \phi(\nu, \mathbf{p})$ and $\phi_{+}(-\bar{P})=\frac{1}{\sqrt{2}} \phi(\nu,-\mathbf{p})$.

\section{THE GAP EQUATION}

In this section, we shall derive the gap equation for the transverse pairing. The starting point is the CJT free energy, which can be approximated in weak coupling as [6, 19, 23.

$$
\Gamma=\Gamma_{n}+\Omega F
$$

where, $\Gamma_{n}$ is the free energy of the normal phase and $F$ the condensation energy density, which is the part of $\Gamma$ responsible to the gap equation. The CJT formula for $F$ reads

$$
F=-\frac{1}{2 \Omega}\left[\operatorname{Tr} \ln D^{-1}-\operatorname{Tr} \ln D_{n}^{-1}-\operatorname{Tr} \ln S^{-1}+\operatorname{Tr} \ln S_{0}^{-1}-\operatorname{Tr}\left(S_{0}^{-1} S-1\right)\right]
$$

where $D$ and $S$ are the full propagators for gluon and quark respectively, $S_{0}$ is the tree level quark propagator and $D_{n}$ is the hard-dense-loop (HDL) gluon propagator. Following the procedure of [24, 25], we approximate

$$
\operatorname{Tr} \ln D^{-1}-\operatorname{Tr} \ln \left[D_{n}^{-1}\right] \simeq \operatorname{Tr}\left[D_{n} \delta \Pi\right]
$$


where

$$
\delta \Pi=\Pi-\Pi_{n}
$$

with $\Pi_{n}$ the HDL resummed gluon self-energy in normal phase. Eq.(26) represents the Hartree-Fock energy of the mean-field theory. In the Coulomb gauge, the HDL gluon propagator is

$$
D_{n, 00}(K)=D_{l}(K), \quad D_{n, 0 i}(K)=D_{n, i 0}(K)=0, \quad D_{n, i j}(K)=\left(\delta_{i j}-\hat{k}_{i} \hat{k}_{j}\right) D_{t}(K)
$$

where $D_{l, t}$ are the longitudinal and transverse propagators respectively and are diagonal in adjoint color space, i.e. $D_{l, t}^{a b}=\delta^{a b} D_{l, t}$. Consequently, we only need the 00 -component, $\Pi^{00}(K)$, and the transverse projection of the ij-components,

$$
\left(\delta_{i j}-\hat{k}_{i} \hat{k}_{j}\right) \Pi^{i j}(K)=\Pi^{i i}(K)-\hat{k}_{i} \hat{k}_{j} \Pi^{i j}(K)
$$

The gluon self-energy in super phase reads

$$
\Pi_{a b}^{\mu \nu}(K)=\frac{1}{2} g^{2} \sum_{P} \operatorname{Tr}\left[\hat{\Gamma}_{a}^{\mu} S(P) \hat{\Gamma}_{b}^{\nu} S\left(P^{\prime}\right)\right]
$$

where $P^{\prime}=P-K, \sum_{P} \equiv T \sum_{\nu} \int \frac{d^{3} \mathbf{p}}{(2 \pi)^{3}}$ and

$$
\hat{\Gamma}_{a}^{\mu} \equiv\left(\begin{array}{cccc}
0 & \sigma^{\mu} T_{a} & 0 & 0 \\
\bar{\sigma}^{\mu} T_{a} & 0 & 0 & 0 \\
0 & 0 & 0 & -\sigma^{\mu} \tilde{T}_{a} \\
0 & 0 & -\bar{\sigma}^{\mu} \tilde{T}_{a} & 0
\end{array}\right)
$$

with $\sigma^{\mu} \equiv(1, \boldsymbol{\sigma}), \bar{\sigma}^{\mu} \equiv(1,-\boldsymbol{\sigma})$, and $T_{a}=\lambda_{a} / 2(a=1, \ldots, 8)$ is the ath $S U(3)$ generator with $\lambda_{a}$ the $a$ th Gell-Mann matrix. Substituting Eq.(21) and (31) into Eq.(30), we have

$$
\begin{aligned}
\Pi_{a b}^{\mu \nu}(K)=\frac{1}{2} g^{2} \sum_{P} \operatorname{Tr} & {\left[\sigma^{\mu} T_{a} S_{21}(P) \sigma^{\nu} T_{b} S_{21}\left(P^{\prime}\right)+\bar{\sigma}^{\mu} T_{a} S_{12}(P) \bar{\sigma}^{\nu} T_{b} S_{12}\left(P^{\prime}\right)\right.} \\
& -\sigma^{\mu} T_{a} S_{23}(P) \sigma^{\nu} \tilde{T}_{b} S_{41}\left(P^{\prime}\right)-\bar{\sigma}^{\mu} T_{a} S_{14}(P) \bar{\sigma}^{\nu} \tilde{T}_{b} S_{32}\left(P^{\prime}\right) \\
& -\sigma^{\mu} \tilde{T}_{a} S_{41}(P) \sigma^{\nu} T_{b} S_{23}\left(P^{\prime}\right)-\bar{\sigma}^{\mu} \tilde{T}_{a} S_{32}(P) \bar{\sigma}^{\nu} T_{b} S_{14}\left(P^{\prime}\right) \\
+ & \left.\sigma^{\mu} \tilde{T}_{a} S_{43}(P) \sigma^{\nu} \tilde{T}_{b} S_{43}\left(P^{\prime}\right)+\bar{\sigma}^{\mu} \tilde{T}_{a} S_{34}(P) \bar{\sigma}^{\nu} \tilde{T}_{b} S_{34}\left(P^{\prime}\right)\right]
\end{aligned}
$$

Substituting Eq.(22) to Eq.(32), we find that

$$
\begin{aligned}
& \operatorname{Tr}\left[\sigma^{\mu} T_{a} S_{21}(P) \sigma^{\nu} T_{b} S_{21}\left(P^{\prime}\right)\right]=\operatorname{Tr}\left[\sigma^{\mu} \frac{1+\boldsymbol{\sigma} \cdot \hat{\mathbf{p}}}{2} \sigma^{\nu} \frac{1+\boldsymbol{\sigma} \cdot \hat{\mathbf{p}}^{\prime}}{2}\right] w_{1}\left(\mathbf{p}, \mathbf{p}^{\prime}\right), \\
& \operatorname{Tr}\left[\bar{\sigma}^{\mu} T_{a} S_{12}(P) \bar{\sigma}^{\nu} T_{b} S_{12}\left(P^{\prime}\right)\right]=\operatorname{Tr}\left[\bar{\sigma}^{\mu} \frac{1-\boldsymbol{\sigma} \cdot \hat{\mathbf{p}}_{2}}{2} \bar{\sigma}^{\nu} \frac{1-\boldsymbol{\sigma} \cdot \hat{\mathbf{p}}^{\prime}}{2}\right] w_{1}^{\prime}\left(\mathbf{p}, \mathbf{p}^{\prime}\right), \\
& \operatorname{Tr}\left[\sigma^{\mu} T_{a} S_{23}(P) \sigma^{\nu} \tilde{T}_{b} S_{41}\left(P^{\prime}\right)\right]=\frac{1}{2} \operatorname{Tr}\left[\sigma^{\mu} \boldsymbol{\sigma} \cdot \hat{\mathbf{e}}_{+}(\hat{\mathbf{p}}) \phi(\nu, \mathbf{p}) \frac{1-\boldsymbol{\sigma} \cdot \hat{\mathbf{p}}}{2} \sigma^{\nu} \boldsymbol{\sigma} \cdot \hat{\mathbf{e}}_{-}\left(\hat{\mathbf{p}}^{\prime}\right) \phi^{*}\left(\nu^{\prime}, \mathbf{p}^{\prime}\right) \frac{1+\boldsymbol{\sigma} \cdot \hat{\mathbf{p}}^{\prime}}{2}\right] w_{2}\left(\mathbf{p}, \mathbf{p}^{\prime}\right), \\
& \operatorname{Tr}\left[\bar{\sigma}^{\mu} T_{a} S_{14}(P) \bar{\sigma}^{\nu} \tilde{T}_{b} S_{32}\left(P^{\prime}\right)\right]=\frac{1}{2} \operatorname{Tr}\left[\bar{\sigma}^{\mu} \boldsymbol{\sigma} \cdot \hat{\mathbf{e}}-(\hat{\mathbf{p}}) \phi(\nu,-\mathbf{p}) \frac{1+\boldsymbol{\sigma} \cdot \hat{\mathbf{p}}}{2} \bar{\sigma}^{\nu} \boldsymbol{\sigma} \cdot \hat{\mathbf{p}}_{+}\left(\hat{\mathbf{p}}^{\prime}\right) \phi^{*}\left(\nu^{\prime},-\mathbf{p}^{\prime}\right) \frac{1-\boldsymbol{\sigma} \cdot \hat{\mathbf{p}}^{\prime}}{2}\right] w_{2}^{\prime}\left(\mathbf{p}, \mathbf{p}^{\prime}\right), \\
& \operatorname{Tr}\left[\sigma^{\mu} \tilde{T}_{a} S_{41}(P) \sigma^{\nu} T_{b} S_{23}\left(P^{\prime}\right)\right]=\frac{1}{2} \operatorname{Tr}\left[\sigma^{\mu} \boldsymbol{\sigma} \cdot \hat{\mathbf{e}}-(\hat{\mathbf{p}}) \phi^{*}(\nu, \mathbf{p}) \frac{1+\boldsymbol{\sigma} \cdot \hat{\mathbf{p}}}{2} \sigma^{\nu} \boldsymbol{\sigma} \cdot \hat{\mathbf{e}}_{+}\left(\hat{\mathbf{p}}^{\prime}\right) \phi\left(\nu^{\prime}, \mathbf{p}^{\prime}\right) \frac{1-\boldsymbol{\sigma} \cdot \hat{\mathbf{p}}^{\prime}}{2}\right] w_{3}\left(\mathbf{p}, \mathbf{p}^{\prime}\right), \\
& \operatorname{Tr}\left[\bar{\sigma}^{\mu} \tilde{T}_{a} S_{32}(P) \bar{\sigma}^{\nu} T_{b} S_{14}\left(P^{\prime}\right)\right]=\frac{1}{2} \operatorname{Tr}\left[\bar{\sigma}^{\mu} \boldsymbol{\sigma} \cdot \hat{\mathbf{e}}_{+}(\hat{\mathbf{p}}) \phi^{*}(\nu,-\mathbf{p}) \frac{1-\boldsymbol{\sigma} \cdot \hat{\mathbf{p}}}{2} \bar{\sigma}^{\nu} \boldsymbol{\sigma} \cdot \hat{\mathbf{e}}_{-}\left(\hat{\mathbf{p}}^{\prime}\right) \phi\left(\nu^{\prime},-\mathbf{p}^{\prime}\right) \frac{1+\boldsymbol{\sigma} \cdot \hat{\mathbf{p}}^{\prime}}{2}\right] w_{3}^{\prime}\left(\mathbf{p}, \mathbf{p}^{\prime}\right), \\
& \operatorname{Tr}\left[\sigma^{\mu} \tilde{T}_{a} S_{43}(P) \sigma^{\nu} \tilde{T}_{b} S_{43}\left(P^{\prime}\right)\right]=\operatorname{Tr}\left[\sigma^{\mu} \frac{1-\boldsymbol{\sigma} \cdot \hat{\mathbf{p}}}{2} \sigma^{\nu} \frac{1-\boldsymbol{\sigma} \cdot \hat{\mathbf{p}}^{\prime}}{2}\right] w_{4}\left(\mathbf{p}, \mathbf{p}^{\prime}\right), \\
& \operatorname{Tr}\left[\bar{\sigma}^{\mu} \tilde{T}_{a} S_{34}(P) \bar{\sigma}^{\nu} \tilde{T}_{b} S_{34}\left(P^{\prime}\right)\right]=\operatorname{Tr}\left[\bar{\sigma}^{\mu} \frac{1+\boldsymbol{\sigma} \cdot \hat{\mathbf{p}}}{2} \bar{\sigma}^{\nu} \frac{1+\boldsymbol{\sigma} \cdot \hat{\mathbf{p}}^{\prime}}{2}\right] w_{4}^{\prime}\left(\mathbf{p}, \mathbf{p}^{\prime}\right)
\end{aligned}
$$


The trace over color space can be readily performed and we have

$$
w_{1,4}\left(\mathbf{p}, \mathbf{p}^{\prime}\right)=w_{1,4}^{\prime}\left(-\mathbf{p},-\mathbf{p}^{\prime}\right)= \begin{cases}\frac{1}{2} \frac{i \nu \pm \xi}{\nu^{2}+\xi^{2}+\Delta^{2}} \frac{i \nu^{\prime} \pm \xi^{\prime}}{\nu^{\prime 2}+\xi^{\prime 2}+\Delta^{\prime 2}}, & a=b=1,2,3 \\ -\frac{1}{4}\left[\frac{1}{i \nu \mp \xi} \frac{i \nu^{\prime} \pm \xi^{\prime}}{\nu^{\prime 2}+\xi^{\prime 2}+\Delta^{\prime 2}}+\frac{1}{\nu^{\prime} \mp \xi^{\prime}} \frac{i \nu \pm \xi}{\nu^{2}+\xi^{2}+\Delta^{2}}\right], & a=b=4, \ldots, 7 \\ \frac{1}{6} \frac{i \nu \pm \xi}{\nu^{2}+\xi^{2}+\Delta^{2}} \frac{i \nu^{\prime} \pm \xi^{\prime}}{\nu^{\prime 2}+\xi^{\prime 2}+\Delta^{\prime 2}}+\frac{1}{3} \frac{1}{i \nu \mp \xi} \frac{1}{i \nu^{\prime} \mp \xi^{\prime}}, & a=b=8\end{cases}
$$

and

$$
w_{2}\left(\mathbf{p}, \mathbf{p}^{\prime}\right)=w_{2}^{\prime}\left(-\mathbf{p},-\mathbf{p}^{\prime}\right)=w_{3}\left(\mathbf{p}, \mathbf{p}^{\prime}\right)=w_{3}^{\prime}\left(-\mathbf{p},-\mathbf{p}^{\prime}\right)= \begin{cases}-\frac{1}{2} \frac{1}{\nu^{2}+\xi^{2}+\Delta^{2}} \frac{1}{\nu^{\prime 2}+\xi^{\prime 2}=\Delta^{\prime 2}}, & a=b=1,2,3 \\ 0, & a=b=4, \ldots, 7 \\ \frac{1}{6} \frac{1}{\nu^{2}+\xi^{2}+\Delta^{2}} \frac{1}{\nu^{\prime 2}+\xi^{\prime 2}+\Delta^{\prime 2}}, & a=b=8\end{cases}
$$

where we have defined $\xi=p-\mu, \Delta^{2}=|\phi(\nu, \mathbf{p})|^{2}$. The \pm on the RHS of Eq.(34) correspond to $w_{1}$ and $w_{4}$ respectively. In weak coupling, the dominant contribution arise from the quasi-particles, therefore we have ignored that from the quasi-antiparticles. The trace over Pauli matrices on the RHS of Eq.(33) are evaluated. We have

$$
\begin{array}{r}
\operatorname{Tr}\left[\sigma^{0} \frac{1 \pm \boldsymbol{\sigma} \cdot \hat{\mathbf{p}}}{2} \sigma^{0} \frac{1 \pm \boldsymbol{\sigma} \cdot \hat{\mathbf{p}}^{\prime}}{2}\right]=\operatorname{Tr}\left[\bar{\sigma}^{0} \frac{1 \pm \boldsymbol{\sigma} \cdot \hat{\mathbf{p}}}{2} \bar{\sigma}^{0} \frac{1 \pm \boldsymbol{\sigma} \cdot \hat{\mathbf{p}}^{\prime}}{2}\right]=\frac{1}{2}\left(1+\hat{\mathbf{p}} \cdot \hat{\mathbf{p}}^{\prime}\right) \\
\operatorname{Tr}\left[\sigma^{i} \frac{1 \pm \boldsymbol{\sigma} \cdot \hat{\mathbf{p}}}{2} \sigma^{i} \frac{1 \pm \boldsymbol{\sigma} \cdot \hat{\mathbf{p}}^{\prime}}{2}\right]=\operatorname{Tr}\left[\bar{\sigma}^{i} \frac{1 \pm \boldsymbol{\sigma} \cdot \hat{\mathbf{p}}}{2} \bar{\sigma}^{i} \frac{1 \pm \boldsymbol{\sigma} \cdot \hat{\mathbf{p}}^{\prime}}{2}\right]=\frac{1}{2}\left(3-\hat{\mathbf{p}} \cdot \hat{\mathbf{p}}^{\prime}\right) \\
\hat{k}_{i} \hat{k}_{j} \operatorname{Tr}\left[\sigma^{i} \frac{1 \pm \boldsymbol{\sigma} \cdot \hat{\mathbf{p}}}{2} \sigma^{j} \frac{1 \pm \boldsymbol{\sigma} \cdot \hat{\mathbf{p}}^{\prime}}{2}\right]=\hat{k}_{i} \hat{k}_{j} \operatorname{Tr}\left[\bar{\sigma}^{i} \frac{1 \pm \boldsymbol{\sigma} \cdot \hat{\mathbf{p}}}{2} \bar{\sigma}^{j} \frac{1 \pm \boldsymbol{\sigma} \cdot \hat{\mathbf{p}}^{\prime}}{2}\right]=\frac{1}{2}\left[1-\hat{\mathbf{p}} \cdot \hat{\mathbf{p}}^{\prime}+2 \hat{\mathbf{k}} \cdot \hat{\mathbf{p}} \hat{\mathbf{k}} \cdot \hat{\mathbf{p}}^{\prime}\right] .
\end{array}
$$

It follows from Eq.(16) that

$$
\boldsymbol{\sigma} \cdot \hat{\mathbf{e}}_{ \pm}(\hat{\mathbf{p}}) \frac{1 \mp \boldsymbol{\sigma} \cdot \hat{\mathbf{p}}}{2}=\hat{\mathbf{e}}_{ \pm}(\hat{\mathbf{p}}) .
$$

and the trace in Eq.(33) is simplified. The terms we need are

$$
\begin{gathered}
\operatorname{Tr}\left[\sigma^{0} \boldsymbol{\sigma} \cdot \hat{\mathbf{e}}_{+}(\hat{\mathbf{p}}) \phi(\nu, \mathbf{p}) \frac{1-\boldsymbol{\sigma} \cdot \hat{\mathbf{p}}}{2} \sigma^{0} \boldsymbol{\sigma} \cdot \hat{\mathbf{e}}_{-}\left(\hat{\mathbf{p}}^{\prime}\right) \phi^{*}\left(\nu^{\prime}, \mathbf{p}^{\prime}\right) \frac{1+\boldsymbol{\sigma} \cdot \hat{\mathbf{p}}^{\prime}}{2}\right]=2 \phi(\nu, \mathbf{p}) \phi^{*}\left(\nu^{\prime}, \mathbf{p}^{\prime}\right) \hat{\mathbf{e}}_{+}(\hat{\mathbf{p}}) \cdot \hat{\mathbf{e}}_{-}\left(\hat{\mathbf{p}}^{\prime}\right) \\
\operatorname{Tr}\left[\sigma^{i} \boldsymbol{\sigma} \cdot \hat{\mathbf{e}}_{+}(\hat{\mathbf{p}}) \phi(\nu, \mathbf{p}) \frac{1-\boldsymbol{\sigma} \cdot \hat{\mathbf{p}}}{2} \sigma^{i} \boldsymbol{\sigma} \cdot \hat{\mathbf{e}}_{-}\left(\hat{\mathbf{p}}^{\prime}\right) \phi^{*}\left(\nu^{\prime}, \mathbf{p}^{\prime}\right) \frac{1+\boldsymbol{\sigma} \cdot \hat{\mathbf{p}}^{\prime}}{2}\right]=-2 \phi(\nu, \mathbf{p}) \phi^{*}\left(\nu^{\prime}, \mathbf{p}^{\prime}\right) \hat{\mathbf{e}}_{+}(\hat{\mathbf{p}}) \cdot \hat{\mathbf{e}}_{-}\left(\hat{\mathbf{p}}^{\prime}\right)
\end{gathered}
$$

and

$$
\begin{aligned}
& \hat{k}_{i} \hat{k}_{j} \operatorname{Tr}\left[\sigma^{i} \boldsymbol{\sigma} \cdot \hat{\mathbf{e}}_{+}(\hat{\mathbf{p}}) \phi(\nu, \mathbf{p}) \frac{1-\boldsymbol{\sigma} \cdot \hat{\mathbf{p}}}{2} \sigma^{j} \boldsymbol{\sigma} \cdot \hat{\mathbf{e}}_{-}\left(\hat{\mathbf{p}}^{\prime}\right) \phi^{*}\left(\nu^{\prime}, \mathbf{p}^{\prime}\right) \frac{1+\boldsymbol{\sigma} \cdot \hat{\mathbf{p}}^{\prime}}{2}\right] \\
= & \frac{4\left(p p^{\prime}-\mathbf{p} \cdot \mathbf{p}^{\prime}\right)}{\left(\mathbf{p}-\mathbf{p}^{\prime}\right)^{2}} \phi(\nu, \mathbf{p}) \phi^{*}\left(\nu^{\prime}, \mathbf{p}^{\prime}\right) \hat{\mathbf{e}}_{+}(\hat{\mathbf{p}}) \cdot \hat{\mathbf{e}}_{-}\left(\hat{\mathbf{p}}^{\prime}\right)-2 \phi(\nu, \mathbf{p}) \phi^{*}\left(\nu^{\prime}, \mathbf{p}^{\prime}\right) \hat{\mathbf{e}}_{+}(\hat{\mathbf{p}}) \cdot \hat{\mathbf{e}}_{-}\left(\hat{\mathbf{p}}^{\prime}\right)
\end{aligned}
$$

The other cases in Eq.(33) can be evaluated along the same lines and we do not write them down explicitly here.

We now turn to the Hartree-Fock energy density in Eq.(26) and divide it into two parts

$$
\Gamma_{H F}=-\frac{1}{2 \Omega} \operatorname{Tr}\left[D_{n} \delta \Pi\right]=\Gamma_{m}+\Gamma_{e}
$$

where the first term comes from magnetic gluons and the second term from electric gluons and we have

$$
\begin{array}{r}
\Gamma_{m}=-\frac{1}{4} g^{2} \sum_{P, P^{\prime}} D_{t}(K)\left(\delta_{i j}-\hat{k}_{i} \hat{k}_{j}\right) \operatorname{Tr}\left[\hat{\Gamma}_{a}^{i} S(P) \hat{\Gamma}_{a}^{j} S\left(P^{\prime}\right)\right] \\
\Gamma_{e}=-\frac{1}{4} g^{2} \sum_{P, P^{\prime}} D_{l}(K) \operatorname{Tr}\left[\hat{\Gamma}_{a}^{0} S(P) \hat{\Gamma}_{a}^{0} S\left(P^{\prime}\right)\right]
\end{array}
$$

As we discussed in our previous paper 19], the net contribution from Eq. (34) and that from the diagonal block of the quark self-energy matrix is to add a wave-function renormalization factor in the gap equation [26, 27, 28, 29], 
which is subleading order. It will not interfere with the angular dependence of the gap function as we have already seen in our previous paper [19]. Therefore, the contribution from Eq.(34) will be ignored and only that from Eq.(35) will be considered here. We find

$$
\begin{aligned}
\Gamma_{m}= & -\frac{2}{3} g^{2} \sum_{P, P^{\prime}} D_{t}(K) \frac{p p^{\prime}-\mathbf{p} \cdot \mathbf{p}^{\prime}}{\left(\mathbf{p}-\mathbf{p}^{\prime}\right)^{2}} \frac{\phi(\nu, \mathbf{p}) \phi^{*}\left(\nu^{\prime}, \mathbf{p}^{\prime}\right) \hat{\mathbf{e}}_{+}(\hat{\mathbf{p}}) \cdot \hat{\mathbf{e}}_{-}\left(\hat{\mathbf{p}}^{\prime}\right)+c . c .}{\left(\nu^{2}+\xi^{2}+\Delta^{2}\right)\left(\nu^{\prime 2}+\xi^{\prime 2}+\Delta^{\prime 2}\right)} \\
& +\left\{\text { a term with } \mathbf{p} \rightarrow-\mathbf{p}, \mathbf{p}^{\prime} \rightarrow-\mathbf{p}^{\prime}\right\}
\end{aligned}
$$

and

$$
\Gamma_{e}=-\frac{1}{3} g^{2} \sum_{P, P^{\prime}} D_{l}(K) \frac{\phi(\nu, \mathbf{p}) \phi^{*}\left(\nu^{\prime}, \mathbf{p}^{\prime}\right) \hat{\mathbf{e}}_{+}(\hat{\mathbf{p}}) \cdot \hat{\mathbf{e}}_{-}\left(\hat{\mathbf{p}}^{\prime}\right)+c . c .}{\left(\nu^{2}+\xi^{2}+\Delta^{2}\right)\left(\nu^{\prime 2}+\xi^{\prime 2}+\Delta^{\prime 2}\right)}+\left\{\text { a term with } \mathbf{p} \rightarrow-\mathbf{p}, \mathbf{p}^{\prime} \rightarrow-\mathbf{p}^{\prime}\right\}
$$

In weak coupling, the approximation $p \simeq p^{\prime} \simeq \mu$ is justified, and we can perform the integral over $\xi$ by picking up the the poles in the diquark propagator. Assuming further that the gap function depends only on the energy and the orientation of the momentum, we find

$$
\Gamma_{H F}=-\frac{1}{6} g^{2} \mu^{4} \int \frac{d \nu}{2 \pi} \int \frac{d \nu^{\prime}}{2 \pi} \int \frac{d^{2} \hat{\mathbf{p}}}{(2 \pi)^{2}} \int \frac{d^{2} \hat{\mathbf{p}}^{\prime}}{(2 \pi)^{2}}\left[D_{t}(K)+D_{l}(K)\right] \frac{\phi(\nu, \mathbf{p}) \phi^{*}\left(\nu^{\prime}, \mathbf{p}^{\prime}\right) \hat{\mathbf{e}}_{+}(\hat{\mathbf{p}}) \cdot \hat{\mathbf{e}}_{-}\left(\hat{\mathbf{p}}^{\prime}\right)+c . c .}{\sqrt{\nu^{2}+\Delta^{2}} \sqrt{\nu^{\prime 2}+\Delta^{\prime 2}}}
$$

In order to evaluate the 3rd and 4th terms on the RHS of Eq.(25), we employ the identities $\operatorname{Tr} \ln S^{-1}=\ln D e t S^{-1}$ and

$$
\operatorname{Det}\left(\begin{array}{cc}
A & B \\
C & D
\end{array}\right)=\operatorname{Det}\left(-C B+C A C^{-1} D\right)=\operatorname{Det}\left(-B C+B D B^{-1} A\right)
$$

with $A, B, C$ and $D$ arbitrary matrices and $B, C$ invertible. We have

$$
\frac{1}{2 \Omega}\left(\operatorname{Tr} \ln S^{-1}-\operatorname{Tr} \ln S_{0}^{-1}\right)=2 \mu^{2} \int \frac{d \nu}{2 \pi} \int \frac{d^{2} \hat{\mathbf{p}}}{(2 \pi)^{2}}\left(|\nu|-\sqrt{\nu^{2}+\Delta^{2}}\right)
$$

The evaluation of the last term on the RHS of Eq.(25) can be readily performed by using the full quark propagator and we end up with

$$
\frac{1}{2 \Omega} \operatorname{Tr}\left(S_{0}^{-1} S-1\right)=2 \mu^{2} \int \frac{d \nu}{2 \pi} \int \frac{d^{2} \hat{\mathbf{p}}}{(2 \pi)^{2}} \frac{\Delta^{2}}{\sqrt{\nu^{2}+\Delta^{2}}}
$$

Therefore, the condensation energy density reads

$$
\begin{aligned}
F= & -\frac{3 \bar{g}^{2} \mu^{4}}{64 \pi^{4}} \int d \nu \int d \nu^{\prime} \int d^{2} \hat{\mathbf{p}} \int d^{2} \hat{\mathbf{p}}^{\prime} V\left(\nu-\nu^{\prime}, \hat{\mathbf{p}} \cdot \hat{\mathbf{p}}^{\prime}\right) \frac{\phi(\nu, \mathbf{p}) \phi^{*}\left(\nu^{\prime}, \mathbf{p}^{\prime}\right) \hat{\mathbf{e}}_{+}(\hat{\mathbf{p}}) \cdot \hat{\mathbf{e}}_{-}\left(\hat{\mathbf{p}}^{\prime}\right)+c . c .}{\sqrt{\nu^{2}+\Delta^{2}} \sqrt{\nu^{\prime 2}+\Delta^{\prime 2}}} \\
& +\frac{\mu^{2}}{4 \pi^{3}} \int d \nu \int d^{2} \hat{\mathbf{p}}\left(|\nu|-\frac{\nu^{2}}{\sqrt{\nu^{2}+\Delta^{2}}}\right)
\end{aligned}
$$

where, the pairing potential $V$ contains the contributions from both magnetic and electric gluons and reads

$$
V\left(\nu-\nu^{\prime}, \hat{\mathbf{p}} \cdot \hat{\mathbf{p}}^{\prime}\right)=D_{t}\left(\nu-\nu^{\prime}, \mathbf{p}-\mathbf{p}^{\prime}\right)+D_{l}\left(\nu-\nu^{\prime}, \mathbf{p}-\hat{\mathbf{p}}^{\prime}\right)
$$

with $\mathbf{p}-\mathbf{p}^{\prime}=\mu\left(\hat{\mathbf{p}}-\hat{\mathbf{p}}^{\prime}\right)$. In the energy-momentum region for Cooper pairing, $\left|\nu-\nu^{\prime}\right|<<\left|\mathbf{p}-\mathbf{p}^{\prime}\right|, D_{t}$ is dominated by Landau damping and $D_{l}$ by Debye screening. We have

$$
V\left(\nu-\nu^{\prime}, \hat{\mathbf{p}} \cdot \hat{\mathbf{p}}^{\prime}\right)=\frac{1}{\left(\mathbf{p}-\mathbf{p}^{\prime}\right)^{2}+\frac{\pi}{4} m_{D}^{2} \frac{\left|\nu-\nu^{\prime}\right|}{\left|\mathbf{p}-\mathbf{p}^{\prime}\right|}}+\frac{1}{\left(\mathbf{p}-\mathbf{p}^{\prime}\right)^{2}+m_{D}^{2}}
$$

where the Debye mass square $m_{D}^{2}=\frac{N_{f} g^{2} \mu^{2}}{2 \pi^{2}}$.

To obtain the gap equation, we introduce the quantity,

$$
\zeta=\frac{\phi}{\sqrt{\nu^{2}+\Delta^{2}}}
$$


The minimization of the condensation energy density with respect to $\zeta$ gives rise to the gap equation

$$
\phi(\nu, \hat{\mathbf{p}})=\frac{3 \bar{g}^{2} \mu^{2}}{4 \pi} \int d \nu^{\prime} \int d^{2} \hat{\mathbf{p}}^{\prime} V\left(\nu-\nu^{\prime}, \hat{\mathbf{p}} \cdot \hat{\mathbf{p}}^{\prime}\right) \frac{\phi\left(\nu^{\prime}, \hat{\mathbf{p}}^{\prime}\right) \hat{\mathbf{e}}_{+}\left(\hat{\mathbf{p}}^{\prime}\right) \cdot \hat{\mathbf{e}}_{-}(\hat{\mathbf{p}})}{\sqrt{\nu^{\prime 2}+\Delta^{\prime 2}}}
$$

The forward singularity corresponds to the divergence of the angular integration when the coupling $g$ in the denominators of (53) is set to zero. To isolate out the singularity, we write the integral on RHS of (55) as

$$
\begin{aligned}
& \int d \nu^{\prime} \int d^{2} \hat{\mathbf{p}}^{\prime} V\left(\nu-\nu^{\prime}, \hat{\mathbf{p}} \cdot \hat{\mathbf{p}}^{\prime}\right) \frac{\phi\left(\nu^{\prime}, \hat{\mathbf{p}}\right)}{\sqrt{\nu^{\prime 2}+\left|\phi\left(\nu^{\prime}, \hat{\mathbf{p}}\right)\right|^{2}}} \\
+ & \int d \nu^{\prime} \int d^{2} \hat{\mathbf{p}}^{\prime} V\left(\nu-\nu^{\prime}, \hat{\mathbf{p}} \cdot \hat{\mathbf{p}}^{\prime}\right)\left[\frac{\phi\left(\nu^{\prime}, \hat{\mathbf{p}}^{\prime}\right) \hat{\mathbf{e}}_{+}\left(\hat{\mathbf{p}}^{\prime}\right) \cdot \hat{\mathbf{e}}_{-}(\hat{\mathbf{p}})}{\sqrt{\nu^{\prime 2}+\left|\phi\left(\nu^{\prime}, \hat{\mathbf{p}}^{\prime}\right)\right|^{2}}}-\frac{\phi\left(\nu^{\prime}, \hat{\mathbf{p}}\right)}{\sqrt{\nu^{\prime 2}+\left|\phi\left(\nu^{\prime}, \hat{\mathbf{p}}\right)\right|^{2}}}\right] .
\end{aligned}
$$

The coupling $g$ in the second integral can be set to zero without introducing divergence. So we did and the gap equation reduces to

$$
\begin{aligned}
\phi(\nu, \hat{\mathbf{p}})= & \int_{-\omega_{0}}^{\omega_{0}} d \nu^{\prime}\left\{\frac{1}{2} \ln \frac{\omega_{c}}{\left|\nu-\nu^{\prime}\right|} \frac{\phi\left(\nu^{\prime}, \hat{\mathbf{p}}\right)}{\sqrt{\nu^{\prime 2}+\left|\phi\left(\nu^{\prime}, \hat{\mathbf{p}}\right)\right|^{2}}}\right. \\
& \left.+\frac{3 \bar{g}^{2}}{4 \pi} \int d^{2} \hat{\mathbf{p}}^{\prime} \frac{1}{1-\hat{\mathbf{p}} \cdot \hat{\mathbf{p}}^{\prime}}\left[\frac{\phi\left(\nu^{\prime}, \hat{\mathbf{p}}^{\prime}\right) \hat{\mathbf{e}}_{+}\left(\hat{\mathbf{p}}^{\prime}\right) \cdot \hat{\mathbf{e}}_{-}(\hat{\mathbf{p}})}{\sqrt{\nu^{\prime 2}+\left|\phi\left(\nu^{\prime}, \hat{\mathbf{p}}^{\prime}\right)\right|^{2}}}-\frac{\phi\left(\nu^{\prime}, \hat{\mathbf{p}}\right)}{\sqrt{\nu^{\prime 2}+\left|\phi\left(\nu^{\prime}, \hat{\mathbf{p}}\right)\right|^{2}}}\right]\right\}
\end{aligned}
$$

where we have carried out the $\hat{\mathbf{p}}^{\prime}$ integral of the first term of (56) and have inintroduced an infrared cutoff $\omega_{0} \sim g \mu$.

\section{THE INTEGRAL EQUATION FOR ANGULAR DEPENDENCE AND ITS SOLUTION}

In the expansion Eq.(19), the circular polarization basis $\hat{\mathbf{e}}_{ \pm}$carry singularity either at the north pole or at the south pole of the spherical coordinates. The coefficient functions that remove this singularity are the Wigner D-functions, instead of the ordinary spherical harmonics. The Winger D-function corresponds to the irreducible representation of the rotation group [30] and is defined by

$$
D_{m m^{\prime}}^{J}(\alpha, \beta, \gamma)=\exp (-i m \alpha) d_{m m^{\prime}}^{J}(\beta) \exp \left(-i m^{\prime} \gamma\right)
$$

with

$$
d_{m m^{\prime}}^{J}(\beta)=<J m\left|e^{-i J_{y} \beta}\right| J m^{\prime}>
$$

Its complex conjugate, $D_{m m^{\prime}}^{J *}(\alpha, \beta, \gamma)$, is the wave function of a symmetric top of definite $J^{2}, J_{z}$ and $\mathbf{J} \cdot \hat{\zeta}$ with $\hat{\zeta}$ the symmetry axis. A diquark pair of opposite helicity of a definite angular momentum represents a parallel case with $\hat{\zeta}$ of the symmetric top replaced by direction of the relative momentum of the pair, $\hat{\mathbf{p}}$. Since $\mathbf{J} \cdot \hat{\mathbf{p}}= \pm 1$, the wave function of a transverse diquark pair ought to be proportional to $D_{m \pm 1}^{J *}$. Here, however, there is a question when carrying this wave function to the diquark pair. A general rotation is defined by three Euler angles $\alpha, \beta, \gamma$ but the gap function depends on only $\hat{\mathbf{p}}$, which is defined by the two polar angles $\varphi$ and $\theta$. In what follows, we shall identify the first two Euler angles with the polar angles, i.e. $\alpha=\varphi, \beta=\theta$, and set $\gamma=0$. As is shown in the appendix B, the real and imaginary parts of $D_{m \pm 1}^{J *}(\varphi, \theta, 0) \hat{\mathbf{e}}_{ \pm}(\hat{\mathbf{p}})$ are proportional to the vector spherical harmonics, which are free from the singularities at $\theta=0$ and $\theta=\pi$. Therefore the partial wave basis of the gap function (23) is $D_{m 1}^{J *}(\varphi, \theta, 0)$.

The partial wave amplitude of the pairing potential, $V\left(\nu-\nu^{\prime}, \hat{\mathbf{p}} \cdot \hat{\mathbf{p}}^{\prime}\right) \hat{\mathbf{e}}_{+}\left(\hat{\mathbf{p}}^{\prime}\right) \cdot \hat{\mathbf{e}}_{-}(\hat{\mathbf{p}})$, is given by the integral

$$
\begin{aligned}
& \int d^{2} \hat{\mathbf{p}} \int d^{2} \hat{\mathbf{p}}^{\prime} D_{m_{1} 1}^{J}(\varphi, \theta, 0) V\left(\nu-\nu^{\prime}, \hat{\mathbf{p}} \cdot \hat{\mathbf{p}}^{\prime}\right) \hat{\mathbf{e}}_{+}\left(\hat{\mathbf{p}}^{\prime}\right) \cdot \hat{\mathbf{e}}_{-}(\hat{\mathbf{p}}) D_{m_{2} 1}^{J *}(\varphi, \theta, 0) \\
= & \frac{\delta_{m_{1} m_{2}}}{2 J+1} \int d^{2} \hat{\mathbf{p}} \int d^{2} \hat{\mathbf{p}}^{\prime} V\left(\nu-\nu^{\prime}, \hat{\mathbf{p}} \cdot \hat{\mathbf{p}}^{\prime}\right) \sum_{m} D_{m 1}^{J}(\varphi, \theta, 0) D_{m 1}^{J *}(\varphi, \theta, 0) \\
= & \frac{\delta_{m_{1} m_{2}}}{2 J+1} \int d^{2} \hat{\mathbf{p}} \int d^{2} \hat{\mathbf{p}}^{\prime} V\left(\nu-\nu^{\prime}, \hat{\mathbf{p}} \cdot \hat{\mathbf{p}}^{\prime}\right) d_{11}^{J}(\Theta) \cos ^{2} \frac{\Theta}{2} \\
= & \frac{8 \pi^{2}}{2 J+1} \delta_{m_{1} m_{2}} \int_{-1}^{1} d \cos \Theta V\left(\nu-\nu^{\prime}, \cos \Theta\right) d_{11}^{J}(\Theta) \cos ^{2} \frac{\Theta}{2}
\end{aligned}
$$


with $\cos \Theta \equiv \hat{\mathbf{p}} \cdot \hat{\mathbf{p}}^{\prime}$, which corresponds to expansion coefficients of $V\left(\nu-\nu^{\prime}, \cos \Theta\right) \cos ^{2} \frac{\Theta}{2}$ in the series of $d_{11}^{J}(\Theta)$ 's. The first equality of (60) follows from the Wigner-Eckart theorem and the second one from the addition formula (B13)(proved in Appendix B)

$$
\sum_{m} D_{m, 1}^{J^{*}}(\varphi, \theta, 0) D_{m, 1}^{J}\left(\varphi^{\prime}, \theta^{\prime}, 0\right) \hat{\mathbf{e}}_{+}(\hat{\mathbf{p}}) \cdot \hat{\mathbf{e}}_{-}\left(\hat{\mathbf{p}}^{\prime}\right)=d_{11}^{J}(\Theta) \cos ^{2} \frac{\Theta}{2}
$$

We have 26, 27, 28]

$$
V\left(\nu-\nu^{\prime}, \hat{\mathbf{p}}-\hat{\mathbf{p}}^{\prime}\right) \cos ^{2} \frac{\Theta}{2}=\frac{1}{6 \mu^{2}} \ln \frac{\omega_{c}}{\left|\nu-\nu^{\prime}\right|} \sum_{J=1}^{\infty}(2 J+1) d_{11}^{J}(\Theta)+\frac{1}{2 \mu^{2}} \sum_{J=1}^{\infty}(2 J+1) c_{J}^{\prime} d_{11}^{J}(\Theta)
$$

where, $\omega_{c}=\frac{1024 \sqrt{2} \pi^{4} \mu}{g^{5}}$ and $c_{J}^{\prime}$ is given by

$$
c_{J}^{\prime}=\int_{-1}^{1} d \cos \Theta \frac{d_{11}^{J}(\Theta) \cos ^{2} \frac{\Theta}{2}-1}{1-\cos \Theta}= \begin{cases}-\frac{3}{2}, & \text { for } J=1 \\ -\sum_{n}^{J} \frac{1}{n}+\frac{1}{2 J(J+1)}, & \text { for } J>1\end{cases}
$$

Unlike the magnitude of the angular momentum, $J$, that is mixed in the solution because of the nonlinearity of the gap equation. The azimuthal quantum number, $J_{z}=m$, remains conserved. For a gap function of a definite $m$,

$$
\phi(\nu, \hat{\mathbf{p}})=\psi_{m}(\nu, \cos \theta) e^{i m \phi}
$$

with $\psi_{m}(\nu, \cos \theta)=$ real, the gap equation (57) implies the following integral equation satisfied by $\psi_{m}(\nu, \cos \theta)$

$$
\begin{aligned}
\psi_{m}(\nu, x)= & \frac{1}{2} \bar{g}^{2} \int_{0}^{\omega_{0}} d \nu^{\prime}\left\{\frac{1}{2}\left(\ln \frac{\omega_{c}}{\left|\nu-\nu^{\prime}\right|}+\ln \frac{\omega_{c}}{\nu+\nu^{\prime}}\right) \frac{\psi_{m}\left(\nu^{\prime}, x\right)}{\sqrt{\nu^{\prime 2}+\psi_{m}^{2}\left(\nu^{\prime}, x\right)}}\right. \\
& \left.+3 \int_{-1}^{1} d x^{\prime} \frac{1}{\left|x-x^{\prime}\right|}\left[\frac{\mathcal{I}_{m}\left(x, x^{\prime}\right) \psi_{m}\left(\nu^{\prime}, x^{\prime}\right)}{\sqrt{\nu^{\prime 2}+\psi_{m}^{2}\left(\nu^{\prime}, x^{\prime}\right)}}-\frac{\psi\left(\nu^{\prime}, x\right)}{\sqrt{\nu^{\prime 2}+\psi_{m}^{2}\left(\nu^{\prime}, x\right)}}\right]\right\},
\end{aligned}
$$

where $x=\cos \theta, x^{\prime}=\cos \theta^{\prime}$ and $\mathcal{I}_{m}\left(x, x^{\prime}\right) \equiv\left|x-x^{\prime}\right| I_{m}\left(x, x^{\prime}\right)$ with

$$
I_{m}\left(x, x^{\prime}\right)=\frac{1}{2 \pi} \int_{0}^{2 \pi} d \varphi \frac{\hat{\mathbf{e}}_{+}\left(\hat{\mathbf{p}}^{\prime}\right) \cdot \hat{\mathbf{e}}_{-}(\hat{\mathbf{p}}) e^{i m\left(\varphi^{\prime}-\varphi\right)}}{1-\hat{\mathbf{p}} \cdot \hat{\mathbf{p}}^{\prime}} .
$$

Substituting the explicit expression of $\hat{\mathbf{e}}_{+}\left(\hat{\mathbf{p}}^{\prime}\right) \cdot \hat{\mathbf{e}}_{-}(\hat{\mathbf{p}})$, we find that $I_{m}\left(x, x^{\prime}\right)$ is real and

$$
I_{-m}\left(x, x^{\prime}\right)=I_{m}\left(-x,-x^{\prime}\right) .
$$

The reduction of the gap equation (65) is parallel to that for the longitudinal pairing case. We shall outline only the main steps here and refer the interested reader to the details in the section IV of [19]. We start with the approximation in 17 ]

$$
\ln \frac{\omega_{c}}{\left|\nu-\nu^{\prime}\right|} \simeq \ln \frac{\omega_{c}}{\left|\nu_{>}\right|}
$$

with $\nu_{>}=\max \left(\nu, \nu^{\prime}\right)$ and introducing the new variable

$$
\xi=\ln \frac{\omega_{c}}{\nu} .
$$

Twice differentiation of Eq. (65) with respect to $\xi$ yields an ordinary differential equation

$$
\frac{d^{2} \psi_{m}(\xi, x)}{d \xi^{2}}+\frac{\bar{g}^{2} \psi_{m}(\xi, x)}{\sqrt{1+\frac{\psi_{m}^{2}(\xi, x)}{\omega_{c}^{2}} e^{2 \xi}}}=0
$$

together with the boundary condition

$$
\frac{d \psi_{m}(\xi, x)}{d \xi}=0
$$


as $\xi \rightarrow \infty$, valid for all $x$. The solution of Eq.(70) up to the subleading order reads:

$$
\psi_{m}(\xi, x)=\Delta_{0} f_{m}(x)[A(\xi, x) u(\xi, x)+B(\xi, x) v(\xi, x)]
$$

where

$$
\begin{array}{r}
u(\xi, x)= \begin{cases}\cos \bar{g}[b(x)-\xi], & \text { for } \xi<b(x) \\
1, & \text { for } \xi>b(x)\end{cases} \\
v(\xi, x)= \begin{cases}-\sin \bar{g}[b(x)-\xi], & \text { for } \xi<b(x) \\
\bar{g} \xi, & \text { for } \xi>b(x)\end{cases}
\end{array}
$$

and

$$
\begin{gathered}
A(\xi, x)=1+\bar{g} \int_{\xi}^{\infty} d \xi^{\prime}\left[\theta\left(b-\xi^{\prime}\right)-\frac{1}{\sqrt{1+u^{2}\left(\xi^{\prime}, x\right) e^{2\left[\xi^{\prime}-b(x)\right]}}}\right] v\left(\xi^{\prime}, x\right) u\left(\xi^{\prime}, x\right) \\
B(\xi, x)=\bar{g} \int_{\xi}^{\infty} d \xi^{\prime}\left[\theta\left(b-\xi^{\prime}\right)-\frac{1}{\left.\sqrt{1+u^{2}\left(\xi^{\prime}, x\right) e^{2\left[\xi^{\prime}-b(x)\right]}}\right] u^{2}\left(\xi^{\prime}, x\right) .}\right.
\end{gathered}
$$

Here, we have introduced the energy gap of $2 \mathrm{SC}$

$$
\Delta_{0}=\frac{2048 \sqrt{2} \pi^{4} \mu}{N_{f}^{5 / 2} g^{5}} e^{-\frac{3 \pi^{2}}{\sqrt{2} g}-\frac{\pi^{2}+4}{8}}
$$

and the quantity $b(x)=\ln \frac{\omega_{c}}{\Delta_{0}\left|f_{m}(x)\right|}$. The angle dependent function $f_{m}(x)$ will be determined below. We notice that at zero Matsubara energy, $\xi \rightarrow \infty, \psi_{m}(0, x)=\Delta_{0} f_{m}(x)$. Substituting Eq.(72) back into Eq.(65), we derive the integral equation for $f_{m}(x)$

$$
f_{m}(x) \ln \left|f_{m}(x)\right|+3 \int_{-1}^{1} d x^{\prime} \frac{1}{\left|x-x^{\prime}\right|}\left[f_{m}(x)-\mathcal{I}_{m}\left(x, x^{\prime}\right) f_{m}\left(x^{\prime}\right)\right]=0 .
$$

The solution of (77) contains all partial waves with $J \geq \max (1,|m|)$ and we shall concentrate on most favored pairing channels, $m=0, \pm 1$. In ${ }^{3} \mathrm{He}, m=0$ refers to the polar phase and $m= \pm 1$ to the A phase. We have

$$
I_{0}\left(x, x^{\prime}\right)=-\frac{1+x x^{\prime}}{2 \sqrt{1-x^{2}} \sqrt{1-x^{\prime 2}}}+\frac{1}{2\left|x-x^{\prime}\right|}\left(\frac{\sqrt{1-x^{\prime 2}}}{\sqrt{1-x^{2}}}+\frac{\sqrt{1-x^{2}}}{\sqrt{1-x^{\prime 2}}}\right)
$$

and

$$
I_{ \pm 1}\left(x, x^{\prime}\right)=\frac{1}{2}\left[\frac{1}{\left|x-x^{\prime}\right|}\left(\frac{1 \pm x^{\prime}}{1 \pm x}+\frac{1 \pm x}{1 \pm x^{\prime}}\right)-\frac{1}{1 \pm x}-\frac{1}{1 \pm x^{\prime}}\right]
$$

Because of the symmetry (67), given a solution of the integral equation of the azimuthal number $m, f_{m}(x)$, the function $f_{m}(-x)$ solves the equation of the azimuthal number $-m$. For the solution starting with the $J=1$ harmonics, we expect that

$$
f_{-m}(x)=f_{m}(-x)
$$

so $f_{0}(x)$ will be an even function of $x$. Since $d_{01}^{J}(\theta)$ is proportional to the associate Legendre function $P_{J}^{1}(x)$, only the partial waves of odd $J$ contribute to $f_{0}(x)$ and the higher harmonics starts from $J=3$. As to $f_{ \pm 1}(x)$, all higher partial waves with $J \geq 2$ will be mixed in, so the free energy reduction is expected to be larger than that of $f_{0}(x)$.

As we have done in [19], this type of integral equation can be solved by a variational method. Upon the leading order solution of the gap function and the definition of angular dependent factor, the condensation energy density Eq.(51) turns out to be a functional of $f(x)$ (see appendix B in [19]). we have

$$
F=\frac{\mu^{2} \Delta_{0}^{2}}{2 \pi^{2}} \mathcal{F}\left[f_{m}\right]
$$


with

$$
\mathcal{F}\left[f_{m}(x)\right]=\int_{-1}^{1} d x f_{m}^{2}(x)\left[\ln \left|f_{m}(x)\right|-\frac{1}{2}\right]+\frac{3}{2} \int_{-1}^{1} d x \int_{-1}^{1} d x^{\prime} \frac{1}{\left|x-x^{\prime}\right|}\left\{f_{m}^{2}(x)-2 f_{m}(x) f_{m}\left(x^{\prime}\right) \mathcal{I}_{m}\left(x, x^{\prime}\right)+f_{m}^{2}\left(x^{\prime}\right)\right\}
$$

It can be easily verified that the variational minimization of Eq.(82) does solve Eq.(77). Before the numerical calculations, we consider a trial function for each case, $m=0, \pm 1$. For $m=0$, the trial function is

$$
f_{0}(x)=c_{0} d_{01}^{1}(\theta)=c_{0} \sqrt{\frac{1-x^{2}}{2}}
$$

and substitute it into the condensation energy density Eq. (82). The minimization yields

$$
c_{0}=\frac{1}{\sqrt{2}} e^{\frac{5}{6}} e^{-\frac{9}{2}}
$$

at which

$$
\mathcal{F}=-1.214 \times 10^{-4}
$$

For $m= \pm 1$, the trial function is

$$
f_{ \pm 1}(x)=c_{ \pm} d_{ \pm 11}^{1}(\cos \theta)=c_{ \pm} \frac{(1 \pm x)}{2}
$$

The same procedure will give rise to

$$
c_{ \pm}=e^{\frac{1}{3}} e^{-\frac{9}{2}}
$$

at which

$$
\mathcal{F}=-8.084 \times 10^{-5}
$$

The target functional corresponding to $m= \pm 1$ has the same value, which is also true when the situation goes to angular momentum mixing. The condensations with $m= \pm 1$, therefore, have the equal priority when the temperature cools down to the critical temperature.

The trial function Eq.(83) and Eq.(86) correspond to what people carried over from the polar phase and A phase of ${ }^{3}$ He respectively [13, 14], which contain p-wave only. The corresponding condensation energy are not optimal as we shall see in the following, which will be lowered further by including higher partial waves.

Following the same procedure in section five in [19], we can obtain the numerical solutions of Eq. (77) and the corresponding condensation energy density. In Fig.1] we show the angular dependence of the gap function for the case $m=0$, the dashed line and the solid one are the trial function and the numerical solution to Eq. (777) respectively. They are depart from each other slightly, however, the condensation energy density corresponding to each case has qualitative difference. We find in this case the minimum of target functional

$$
\mathcal{F}=-1.236 \times 10^{-4}
$$

which drop from Eq.85) by 1.81 percent.

The same is true in the case $m= \pm 1$. In this case, the trial functions (dashed lines) and the numerical solutions to Eq.(77) (solid lines) have been plotted in Fig.2. We find the minimum of the target functional corresponding to solid lines

$$
\mathcal{F}=-8.494 \times 10^{-5}
$$

which drop from Eq. (88) by 5.07 percent.

As we have found in [19], the drop of the condensation energy with longitudinal pairing by angular momentum mixing is a small amount. Here, with transverse pairing, the situation is also true. With these small amount drop in condensation energy, the non-spherical pairing of the polar and A-phases in single flavor CSC remains not energetically more favored than the spherical pairing state CSL.

It is instructive to examine the angular momentum content of our solutions according to

$$
f_{m}(\cos \theta)=\sum_{J=1}^{\infty}(2 J+1) b_{J} d_{m 1}^{J}(\theta)
$$




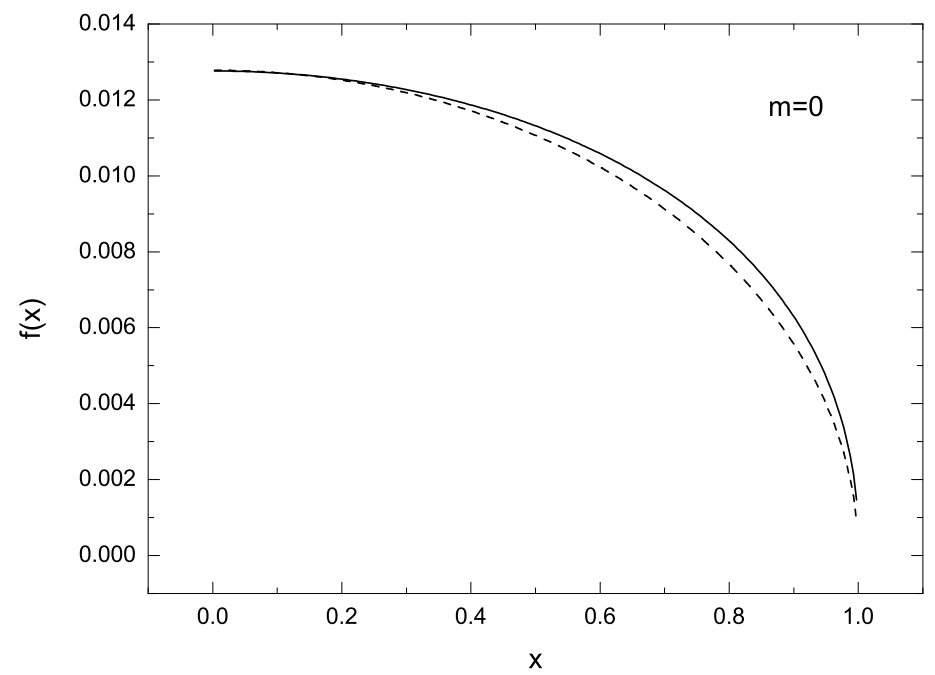

FIG. 1: The angular dependence of the gap function for $\mathrm{m}=0$. The dashed and the solid lines correspond to trial function Eq.(83) and the numerical solution to Eq.(77) respectively.

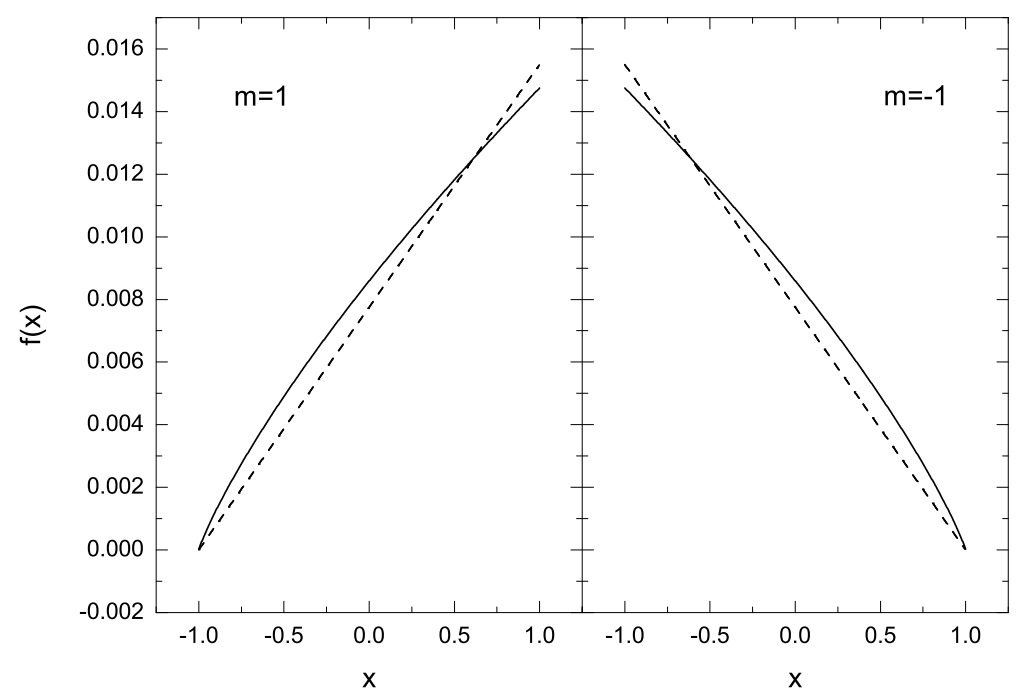

FIG. 2: The angular dependence of the gap function for $m= \pm 1$. The dashed and the solid lines correspond to trial function Eq.(86) and the numerical solution to Eq.(77) respectively.

for $m=0, \pm 1$, we found

$$
f_{0}(\cos \theta)=\frac{1}{\sqrt{2}} e^{5 / 6} e^{-9 / 2}\left[1.0085 d_{01}^{1}(\theta)+0.0426 d_{01}^{3}(\theta)+\ldots\right]
$$

and

$$
f_{ \pm 1}(\cos \theta)=e^{1 / 3} e^{-9 / 2}\left[1.0269 d_{ \pm 11}^{1}(\theta) \mp 0.0943 d_{ \pm 11}^{2}(\theta)+\ldots\right]
$$


TABLE I: The condensation energy of various non-spherical pairing states in unit of the transverse CSL condensation energy with or without angular momentum mixing.

\begin{tabular}{ccccc}
\hline \hline & \multicolumn{2}{c}{ Without mixing } & \multicolumn{2}{c}{ With mixing } \\
$F / F_{C S L}$ & Longitudinal & Transverse & Longitudinal & Transverse \\
\hline Polar & $0.65 e^{-12} / e^{-9}$ & 0.88 & $0.67 e^{-12} / e^{-9}$ & 0.9 \\
$\mathrm{~A}$ & $0.88 e^{-12} / e^{-9}$ & 0.65 & $0.9 e^{-12} / e^{-9}$ & 0.68 \\
\hline \hline
\end{tabular}

\section{SUMMARY AND DISCUSSIONS}

If the pairing potential extends to more than one partial waves, a non-spherical energy gap cannot be restricted within a single angular momentum channel because of the nonlinearity of the gap equation. The corresponding free energy will be brought down by the mixing. In case of the color superconductivity mediated by one-gluon exchange, the pairing potential mediated by one-gluon exchange for all partial waves equal in leading order in QCD running coupling constant. The angular momentum mixing will occur to the leading order of the gap function and free energy of all non-spherical pairing states in the literature needs to be recalculated. So we examined the angular momentum mixing in this paper for transverse pairing and in a previous paper for longitudinal pairing. We derived the nonlinear integral equation for the angular dependence of the gap function and solved it numerically. We found that the effect is numerically small, which may be attributed to the descending pairing strength of higher partial waves beyond the leading order. Our results on the condensate energy of various non-spherical pairing states of single quark flavor are listed in the table I below, in which the ratios without mixing are also listed for comparison.

While the free energy of all non-spherical pairing states are lowered by the angular momentum mixing, the amount of the drop is too small to beat the spherical CSL state of transverse pairing. The energy balance between the CSL and non-spherical states may be offset by anisotropy or the violation of time reversal invariance. In a compact star, the magnetic field and the stellar revolution provides such a circumstance. The magnetic field, however, is unlikely to discriminate the non-spherical pairing states from the spherical ones in the bulk because of the Meissner effect in the CSC phase [16]. In addition, it suppresses all single flavor pairing because of the critical field. As to the stellar revolution, the optimal partition of the angular momentum into the motion of the center of mass and the spin of Cooper pairs remains to be determined. But the non-spherical condensate will response differently from the spherical ones because of the preferred direction in the former.

The slightly complicated non-spherical CSC phase, the transverse planar phase exhibits high potential to be able to compete CSL phase since the gain of the condensation energy from the former to the latter is only 2 percent 14 , falling within the range of the percentage increment of the condensation energy because of the angular momentum mixing discovered so far. Technically, the color structure of the order parameter involves two antisymmetric GellMann matrices and the present formulation needs to be generalized. This is currently investigated and the result will be reported shortly.

\section{Acknowledgments}

We would like to extend our gratitude to D. Rischke, T. Schäfer, A. Schmitt for stimulating discussions. We are also benefitted from conversations with M. Huang, J.R. Li, Q. wang and P.F. Zhuang. The work of D. F. H. and H. C. R. is supported in part by NSFC under grant Nos. 10575043, 10735040. The work of D. F. H. is also supported in part by Educational Committee under under grants NCET-05-0675 project No. IRT0624.

\section{APPENDIX A: THE ENTRIES OF THE FULL QUARK PROPAGATOR}

In order to obtain the full quark propagator, we introduce

$$
\Gamma_{0}=\left(\begin{array}{cc}
\gamma_{0} & 0 \\
0 & \gamma_{0}
\end{array}\right) \quad \text { and } \quad \mathrm{V}=\left(\begin{array}{llll}
1 & 0 & 0 & 0 \\
0 & 0 & 1 & 0 \\
0 & 1 & 0 & 0 \\
0 & 0 & 0 & 1
\end{array}\right)
$$


Using these two matrices, we can transform the inverse quark propagator into a block diagonal form

$$
G(P)=V S^{-1}(P) \Gamma_{0} V^{-1}=\left(\begin{array}{cc}
T^{-1}\left(P, \lambda_{2}\right) & 0 \\
0 & T^{-1}\left(-P,-\lambda_{2}\right)
\end{array}\right)
$$

where the matrix $T$ is given by

$$
T\left(P, \lambda_{2}\right)=\left(\begin{array}{cc}
i \nu+\mu-\boldsymbol{\sigma} \cdot \mathbf{p} & \lambda_{2} \boldsymbol{\sigma} \cdot \boldsymbol{\Psi}(P) \\
\lambda_{2} \boldsymbol{\sigma} \cdot \boldsymbol{\Psi}^{*}(\bar{P}) & i \nu-\mu-\boldsymbol{\sigma} \cdot \mathbf{p}
\end{array}\right)
$$

and can be inverted readily by using the projective operators $P_{ \pm}=(1 \pm \boldsymbol{\sigma} \cdot \hat{\mathbf{p}}) / 2$. Then we have the full quark propagator

$$
S(P)=\Gamma_{0} V^{-1} G^{-1}(P) V=\left(\begin{array}{cccc}
S_{11} & S_{12} & S_{13} & S_{14} \\
S_{21} & S_{22} & S_{23} & S_{24} \\
S_{31} & S_{32} & S_{33} & S_{34} \\
S_{41} & S_{42} & S_{43} & S_{44}
\end{array}\right)
$$

with each entry given by

$$
\begin{aligned}
& S_{11}=S_{13}=S_{22}=S_{24}=S_{31}=S_{33}=S_{42}=S_{44}=0 \\
& S_{12}=-\sum_{e \pm} \frac{i \nu-e(p+e \mu)}{\nu^{2}+(p+e \mu)^{2}+\lambda_{2}^{2} \boldsymbol{\sigma} \cdot \boldsymbol{\Psi}(-P) \boldsymbol{\sigma} \cdot \mathbf{\Psi}^{*}(-\bar{P})} \frac{1+e \boldsymbol{\sigma} \cdot \hat{\mathbf{p}}}{2} \\
& S_{14}=-\lambda_{2} \boldsymbol{\sigma} \cdot \boldsymbol{\Psi}(-P) \sum_{e \pm} \frac{1}{\nu^{2}+(p-e \mu)^{2}+\lambda_{2}^{2} \boldsymbol{\sigma} \cdot \mathbf{\Psi}^{*}(-\bar{P}) \boldsymbol{\sigma} \cdot \boldsymbol{\Psi}(-P)} \frac{1+e \boldsymbol{\sigma} \cdot \hat{\mathbf{p}}}{2} \\
& S_{21}=-\sum_{e \pm} \frac{i \nu+e(p-e \mu)}{\nu^{2}+(p-e \mu)^{2}+\lambda_{2}^{2} \boldsymbol{\sigma} \cdot \boldsymbol{\Psi}(P) \boldsymbol{\sigma} \cdot \mathbf{\Psi}^{*}(\bar{P})} \frac{1+e \boldsymbol{\sigma} \cdot \hat{\mathbf{p}}}{2} \\
& S_{23}=\lambda_{2} \boldsymbol{\sigma} \cdot \boldsymbol{\Psi}(P) \sum_{e \pm} \frac{1}{\nu^{2}+(p+e \mu)^{2}+\lambda_{2}^{2} \boldsymbol{\sigma} \cdot \mathbf{\Psi}^{*}(\bar{P}) \boldsymbol{\sigma} \cdot \boldsymbol{\Psi}(P)} \frac{1+e \boldsymbol{\sigma} \cdot \hat{\mathbf{p}}}{2} \\
& S_{32}=-\lambda_{2} \boldsymbol{\sigma} \cdot \mathbf{\Psi}^{*}(-\bar{P}) \sum_{e \pm} \frac{1}{\nu^{2}+(p+e \mu)^{2}+\lambda_{2}^{2} \boldsymbol{\sigma} \cdot \boldsymbol{\Psi}(-P) \boldsymbol{\sigma} \cdot \mathbf{\Psi}^{*}(-\bar{P})} \frac{1+e \boldsymbol{\sigma} \cdot \hat{\mathbf{p}}}{2} \\
& S_{34}=-\sum_{e \pm} \frac{i \nu+e(p-e \mu)}{\nu^{2}+(p-e \mu)^{2}+\lambda_{2}^{2} \boldsymbol{\sigma} \cdot \boldsymbol{\Psi}^{*}(-\bar{P}) \boldsymbol{\sigma} \cdot \boldsymbol{\Psi}(-P)} \frac{1+e \boldsymbol{\sigma} \cdot \hat{\mathbf{p}}}{2} \\
& S_{41}=\lambda_{2} \boldsymbol{\sigma} \cdot \Psi^{*}(\bar{P}) \sum_{e \pm} \frac{1}{\nu^{2}+(p-e \mu)^{2}+\lambda_{2}^{2} \boldsymbol{\sigma} \cdot \boldsymbol{\Psi}(P) \boldsymbol{\sigma} \cdot \mathbf{\Psi}^{*}(\bar{P})} \frac{1+e \boldsymbol{\sigma} \cdot \hat{\mathbf{p}}}{2} \\
& S_{43}=-\sum_{e \pm} \frac{i \nu+e(p+e \mu)}{\nu^{2}+(p+e \mu)^{2}+\lambda_{2}^{2} \boldsymbol{\sigma} \cdot \Psi^{*}(\bar{P}) \boldsymbol{\sigma} \cdot \boldsymbol{\Psi}(P)} \frac{1+e \boldsymbol{\sigma} \cdot \hat{\mathbf{p}}}{2}
\end{aligned}
$$

In order to get rid of the Pauli matrices in the denominators of the propagator in Eq. A5 , we expanded $\boldsymbol{\Psi}$ in terms of the two circular polarization basis as Eq.(19). We have

$$
\begin{aligned}
\boldsymbol{\sigma} \cdot \boldsymbol{\Psi}(P) \boldsymbol{\sigma} \cdot \boldsymbol{\Psi}^{*}(\bar{P}) & =\boldsymbol{\Psi}(P) \cdot \boldsymbol{\Psi}^{*}(\bar{P})+i \boldsymbol{\sigma} \cdot\left[\boldsymbol{\Psi}(P) \times \boldsymbol{\Psi}^{*}(\bar{P})\right] \\
& =(1+\boldsymbol{\sigma} \cdot \hat{\mathbf{p}}) \phi_{+}(P) \phi_{+}^{*}(\bar{P})+(1-\boldsymbol{\sigma} \cdot \hat{\mathbf{p}}) \phi_{-}(P) \phi_{-}^{*}(\bar{P})
\end{aligned}
$$

Therefore, the entry Eqs. $\mathrm{A} 5 \mathrm{~d}$ ) can be written as

$$
\begin{aligned}
S_{21}= & -\frac{i \nu-(p+\mu)}{\nu^{2}+(p+\mu)^{2}+\lambda_{2}^{2}\left[(1+\boldsymbol{\sigma} \cdot \hat{\mathbf{p}}) \phi_{+}(P) \phi_{+}^{*}(\bar{P})+(1-\boldsymbol{\sigma} \cdot \hat{\mathbf{p}}) \phi_{-}(P) \phi_{-}^{*}(\bar{P})\right]} \frac{1-\boldsymbol{\sigma} \cdot \hat{\mathbf{p}}}{2} \\
& -\frac{i \nu+(p-\mu)}{\nu^{2}+(p-\mu)^{2}+\lambda_{2}^{2}\left[(1+\boldsymbol{\sigma} \cdot \hat{\mathbf{p}}) \phi_{+}(P) \phi_{+}^{*}(\bar{P})+(1-\boldsymbol{\sigma} \cdot \hat{\mathbf{p}}) \phi_{-}(P) \phi_{-}^{*}(\bar{P})\right]} \frac{1+\boldsymbol{\sigma} \cdot \hat{\mathbf{p}}}{2}
\end{aligned}
$$

It can be shown that

$$
f(\boldsymbol{\sigma} \cdot \hat{\mathbf{p}}) \frac{1 \pm \boldsymbol{\sigma} \cdot \hat{\mathbf{p}}}{2}=f( \pm 1) \frac{1 \pm \boldsymbol{\sigma} \cdot \hat{\mathbf{p}}}{2}
$$


with $f(\boldsymbol{\sigma} \cdot \hat{\mathbf{p}})$ is an arbitrary function of $\boldsymbol{\sigma} \cdot \hat{\mathbf{p}}$ as long as $f( \pm 1)$ is well-defined. Using this identity, we find

$$
S_{21}=-\frac{i \nu-(p+\mu)}{\nu^{2}+(p+\mu)^{2}+2 \lambda_{2}^{2} \phi_{-}(P) \phi_{-}^{*}(\bar{P})} \frac{1-\boldsymbol{\sigma} \cdot \hat{\mathbf{p}}}{2}-\frac{i \nu+(p-\mu)}{\nu^{2}+(p-\mu)^{2}+2 \lambda_{2}^{2} \phi_{+}(P) \phi_{+}^{*}(\bar{P})} \frac{1+\boldsymbol{\sigma} \cdot \hat{\mathbf{p}}}{2}
$$

The other entries of the quark propagator can be simplified along the same lines. Furthermore the entries with $\Psi$ or $\boldsymbol{\Psi}^{*}$ in the numerators can be reduced following the identity (39). The final expression of the quark propagator is presented at the end of Sec.II and is used to calculate the condensation energy density in Eq.(25).

\section{APPENDIX B: VECTOR SPHERICAL HARMONICS AND ADDITION FORMULA}

To each momentum p, one defines two transverse spherical harmonics [31]:

$$
\mathbf{Y}_{j m}^{(1)}(\hat{\mathbf{p}})=\mathbf{X}_{j m}(\hat{\mathbf{p}})=\frac{1}{\sqrt{j(j+1)}} \mathbf{L} Y_{j m}(\hat{\mathbf{p}})
$$

and

$$
\mathbf{Y}_{j m}^{(2)}(\hat{\mathbf{p}})=\hat{\mathbf{p}} \times \mathbf{X}_{j m}(\hat{\mathbf{p}})
$$

where the angular momentum operator $\mathbf{L}=-i \mathbf{p} \times \nabla_{\mathbf{p}}$ with $\nabla_{\mathbf{p}}$ the gradient operator with respect to $\mathbf{p}$. It follows from the relation bewteen the scalar spherical harmonics and the Wigner D-function that

$$
\mathbf{X}_{j m}(\hat{\mathbf{p}})=\sqrt{\frac{2 j+1}{4 \pi j(j+1)}}<j m\left|\mathbf{J} e^{-i J_{z} \varphi} e^{-i J_{y} \theta}\right| j 0>^{*}
$$

It is straightforward to show that

$$
\hat{\mathbf{e}}_{ \pm}^{*}(\hat{\mathbf{p}}) \cdot \mathbf{J} e^{-i J_{z} \varphi} e^{-i J_{y} \theta}=\frac{1}{\sqrt{2}} e^{-i J_{z} \varphi} e^{-i J_{y} \theta} J_{ \pm}
$$

with $J_{ \pm}=J_{x} \pm i J_{y}$ the raising and lowering operators. Therefore

$$
\hat{\mathbf{e}}_{ \pm}^{*}(\hat{\mathbf{p}}) \cdot \mathbf{X}_{j m}(\hat{\mathbf{p}})=\sqrt{\frac{2 j+1}{8 \pi}} D_{m \pm 1}^{j *}(\varphi, \theta, 0)
$$

and we have

$$
\mathbf{Y}_{j m}^{(1)}(\hat{\mathbf{p}})=\mathbf{X}_{j m}(\hat{\mathbf{p}})=\sqrt{\frac{2 j+1}{8 \pi}}\left[D_{m 1}^{j^{*}}(\varphi, \theta, 0) \hat{\mathbf{e}}_{+}(\hat{\mathbf{p}})+D_{m-1}^{j^{*}}(\varphi, \theta, 0) \hat{\mathbf{e}}_{-}(\hat{\mathbf{p}})\right]
$$

and

$$
\mathbf{Y}_{j m}^{(2)}(\hat{\mathbf{p}})=\hat{\mathbf{p}} \times \mathbf{X}_{j m}(\hat{\mathbf{p}})=-i \sqrt{\frac{2 j+1}{8 \pi}}\left[D_{m 1}^{j^{*}}(\varphi, \theta, 0) \hat{\mathbf{e}}_{+}(\hat{\mathbf{p}})-D_{m-1}^{j^{*}}(\varphi, \theta, 0) \hat{\mathbf{e}}_{-}(\hat{\mathbf{p}})\right]
$$

Both $\mathbf{Y}_{j m}^{(1)}(\hat{\mathbf{p}})$ and $\mathbf{Y}_{j m}^{(2)}(\hat{\mathbf{p}})$ are regular at poles $\theta=0$ and $\theta=\pi$, so are $D_{m 1}^{j^{*}}(\varphi, \theta, 0) \hat{\mathbf{e}}_{+}(\hat{\mathbf{p}})$ and $D_{m-1}^{j^{*}}(\varphi, \theta, 0) \hat{\mathbf{e}}_{-}(\hat{\mathbf{p}})$.

In order to obtain the addition formula for Wigner D-function, we need to know the Euler angles of the product of two rotations with Euler angles $(\varphi, \theta, 0)$ and $\left(\varphi^{\prime}, \theta^{\prime}, 0\right)$. The composition rule of the transformation parameters of a Lie group is independent of the representations. For the rotation group, the $J=1 / 2$ representation is the most convenient one to extract the composition rule. For $J=1 / 2$, we have

$$
D^{\frac{1}{2}}(\alpha, \beta, \gamma)=e^{-\frac{i}{2} \sigma_{3} \alpha} e^{-\frac{i}{2} \sigma_{2} \beta} e^{-\frac{i}{2} \sigma_{3} \gamma}
$$

with $\sigma_{2,3}$ the Pauli matrices. We denote the combined rotation

$$
D^{\frac{1}{2}}(\varphi, \theta, 0) D^{\frac{1}{2}}\left(\varphi^{\prime}, \theta^{\prime}, 0\right)=D^{\frac{1}{2}}(\alpha, \beta, \gamma)
$$


with $\alpha, \beta, \gamma$ the Euler angles of the combined rotation. Using Eq.(B8), we obtain

$$
\begin{aligned}
& \left(\begin{array}{cc}
\cos \frac{\beta}{2} e^{-\frac{i}{2}(\alpha+\gamma)} & -\sin \frac{\beta}{2} e^{-\frac{i}{2}(\alpha-\gamma)} \\
\sin \frac{\beta}{2} e^{\frac{i}{2}(\alpha-\gamma)} & \cos \frac{\beta}{2} e^{\frac{i}{2}(\alpha+\gamma)}
\end{array}\right) \\
= & \left(\begin{array}{cc}
\cos \frac{\theta}{2} \cos \frac{\theta^{\prime}}{2} e^{\frac{i}{2}\left(\varphi-\varphi^{\prime}\right)}+\sin \frac{\theta}{2} \sin \frac{\theta^{\prime}}{2} e^{-\frac{i}{2}\left(\varphi-\varphi^{\prime}\right)} & -\cos \frac{\theta}{2} \sin \frac{\theta^{\prime}}{2} e^{\frac{i}{2}\left(\varphi-\varphi^{\prime}\right)}+\sin \frac{\theta}{2} \cos \frac{\theta^{\prime}}{2} e^{-\frac{i}{2}\left(\varphi-\varphi^{\prime}\right)} \\
-\sin \frac{\theta}{2} \cos \frac{\theta^{\prime}}{2} e^{\frac{i}{2}\left(\varphi-\varphi^{\prime}\right)}+\cos \frac{\theta}{2} \sin \frac{\theta^{\prime}}{2} e^{-\frac{i}{2}\left(\varphi-\varphi^{\prime}\right)} & \sin \frac{\theta}{2} \sin \frac{\theta^{\prime}}{2} e^{\frac{i}{2}\left(\varphi-\varphi^{\prime}\right)}+\cos \frac{\theta}{2} \cos \frac{\theta^{\prime}}{2} e^{-\frac{i}{2}\left(\varphi-\varphi^{\prime}\right)}
\end{array}\right)
\end{aligned}
$$

The equivalence of each block on both sides gives rise to

$$
\cos \beta=\cos \theta \cos \theta^{\prime}+\sin \theta \sin \theta^{\prime} \cos \left(\varphi-\varphi^{\prime}\right)=\hat{\mathbf{p}} \cdot \hat{\mathbf{p}}^{\prime}
$$

and

$$
\begin{aligned}
e^{i(\alpha+\gamma)} \cos ^{2} \frac{\beta}{2} & =\hat{\mathbf{e}}_{+}(\hat{\mathbf{p}}) \cdot \hat{\mathbf{e}}_{-}\left(\hat{\mathbf{p}}^{\prime}\right) \\
e^{i(\alpha-\gamma)} \sin ^{2} \frac{\beta}{2} & =-\hat{\mathbf{e}}_{+}(\hat{\mathbf{p}}) \cdot \hat{\mathbf{e}}_{+}\left(\hat{\mathbf{p}}^{\prime}\right) \\
e^{-i(\alpha-\gamma)} \sin ^{2} \frac{\beta}{2} & =-\hat{\mathbf{e}}_{-}(\hat{\mathbf{p}}) \cdot \hat{\mathbf{e}}_{-}\left(\hat{\mathbf{p}}^{\prime}\right) \\
e^{-i(\alpha+\gamma)} \cos ^{2} \frac{\beta}{2} & =\hat{\mathbf{e}}_{-}(\hat{\mathbf{p}}) \cdot \hat{\mathbf{e}}_{+}\left(\hat{\mathbf{p}}^{\prime}\right)
\end{aligned}
$$

These equations fix on the Euler angles of the combined rotation. Therefore, the composition rule of two rotations for representation of arbitrary $\mathrm{J}$ reads

$$
\sum_{m} D_{m, 1}^{J^{*}}(\varphi, \theta, 0) D_{m, 1}^{J}\left(\varphi^{\prime}, \theta^{\prime}, 0\right)=D_{11}^{J}(\alpha, \beta, \gamma)=\frac{d_{11}^{J}(\beta) \cos ^{2} \frac{\beta}{2}}{\hat{\mathbf{e}}_{+}(\hat{\mathbf{p}}) \cdot \hat{\mathbf{e}}_{-}\left(\hat{\mathbf{p}}^{\prime}\right)}
$$

[1] B. Barrois, Nucl. Phys. B129, 390(1977); S. C. Frautschi, in Hadronic matter at extreme energy density, edited by N. Cabibbo and L. Sertorio (Plenum Press, 1980).

[2] D. Bailin, and A. Love, Phys. Rep. 107, 325 (1984).

[3] M. Alford, K. Rajagopal and F. Wilczek, Phys. Lett. B422, 247 (1998).

[4] R. Rapp, T. Schäfer, E. V. Shuryak, and M. Velkovsky, Phys. Rev. Lett. 81, 53 (1998).

[5] M. Alford, K. Rajagopal, and F. Wilczek, Nucl. Phys. B537, 443 (1999).

[6] Dirk H. Rischke, Prog. Part. Nucl. Phys. 52:197-296, 2004.

[7] M. G. Alford, A. Schmitt, K, Rajagopal and T. Schäfer, arXiv: 0709,4635[hep-ph].

[8] Mei Huang, Pengfei Zhuang and Weiqin Chao, Phys. Rev. D67, 065015 (2003).

[9] I. Shovkovy and M. Huang, Phys. Lett. B564, 205 (2003).

[10] M. Alford, C. Kouvaris, and K. Rajagopal, Phys. Rev. Lett. 92, 222001 (2004).

[11] M. G. Alford, J. A. Bowers and K. Rajagopal, Phys. Rev. D63, 074061 (2001).

[12] P. F. Befaque, H. Caldas and G. Rupak, Phys. Rev. Lett. 91, 247002 (2003).

[13] T. Schäfer, Phys. Rev. D62, 094007 (2000).

[14] A. Schmitt, Phys. Rev. D71, 054016 (2005); nucl-th/0405076 (Ph. D. thesis).

[15] A. Schmitt, I. A. Shovkovy and Q. Wang, Phys. Rev. Lett. 94211101 (2005).

[16] A. Schmitt, Q. Wang and D. H. Rischke, Phys. Rev. Lett. 91242301 (2003).

[17] D. T. Son, Phys. Rev. D59, 094019 (1999).

[18] R. D. Pisarski, and D. H. Rischke, Phys. Rev. D61, 074017 (2000).

[19] B. Feng, D-F. Hou, and H-C. Ren, Nucl. Phys. B796, 500 (2008).

[20] B. Feng, D-F. Hou, and H-C. Ren, to be published.

[21] R. D. Pisarski, and D. H. Rischke, Phys. Rev. D60, 094013 (1999).

[22] B. Feng, D-F. Hou, J-R. Li, and H-C. Ren, Nucl. Phys. B754, 351 (2006).

[23] J. M. Cornwall, R. Jackiw, and E. Tomboulis, Phys. Rev. D10, 2428 (1974).

[24] I. Giannakis, D-F. Hou, H-C. Ren, and D. H. Rischke, Phys. Rev. Lett. 93, 232301 (2004).

[25] J. I. Noronha, H-C. Ren, I. Giannakis, D-F. Hou, and D. H. Rischke, Phys. Rev. D73, 094009 (2006).

[26] W. E. Brown, J. T. Liu, and H-C. Ren, Phys. Rev. D61, 114012 (2000).

[27] W. E. Brown, J. T. Liu, and H-C. Ren, Phys. Rev. D62, 054016 (2000).

[28] W. E. Brown, J. T. Liu, and H-C. Ren, Phys. Rev. D62, 054013 (2000).

[29] Qun Wang and Dirk H. Rischke, Phys. Rev. D65, 054005 (2002).

[30] J-Y. Zeng, Quantum mechanics, Science publishing company, China, 2000, volume II, Chapter 6.

[31] J. D. Jackson, Classical Electrodynamics, John Wiley and Sons, Inc., 1975, Chapter 16. 